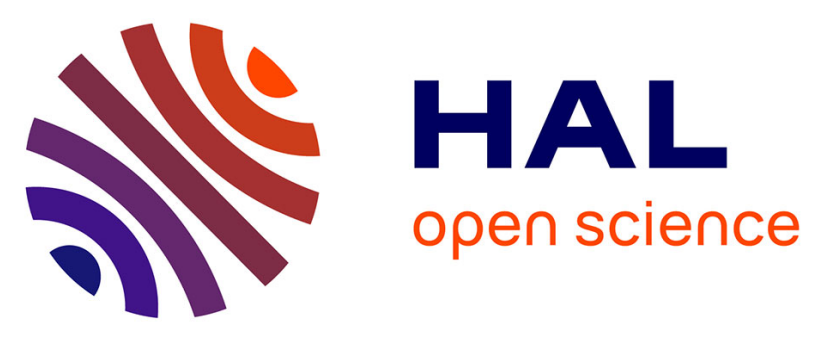

\title{
A methodology to calibrate and to validate effective solid potentials of heterogeneous porous media from computed tomography scans and laboratory-measured nanoindentation data
}

\author{
Siavash Monfared, Hadrien Laubie, Farhang Radjai, Mija Hubler, Roland \\ Pellenq, Franz-Josef Ulm
}

\section{To cite this version:}

Siavash Monfared, Hadrien Laubie, Farhang Radjai, Mija Hubler, Roland Pellenq, et al.. A methodology to calibrate and to validate effective solid potentials of heterogeneous porous media from computed tomography scans and laboratory-measured nanoindentation data. Acta Geotechnica, 2018, 13 (6), pp.1369-1394. 10.1007/s11440-018-0687-9 . hal-01958690

\author{
HAL Id: hal-01958690 \\ https://hal.science/hal-01958690
}

Submitted on 21 Oct 2019

HAL is a multi-disciplinary open access archive for the deposit and dissemination of scientific research documents, whether they are published or not. The documents may come from teaching and research institutions in France or abroad, or from public or private research centers.
L'archive ouverte pluridisciplinaire HAL, est destinée au dépôt et à la diffusion de documents scientifiques de niveau recherche, publiés ou non, émanant des établissements d'enseignement et de recherche français ou étrangers, des laboratoires publics ou privés. 


\title{
A methodology to calibrate and to validate effective solid potentials of heterogeneous porous media from computed tomography scans and laboratory-measured nanoindentation data
}

\author{
Siavash Monfared ${ }^{1}$ (i) $\cdot$ Hadrien Laubie $^{1} \cdot$ Farhang Radjai $^{2,3} \cdot$ Mija Hubler $^{4} \cdot$ Roland Pellenq $^{2,5} \cdot$ Franz-Josef Ulm $^{1}$
}

\begin{abstract}
Built on the framework of effective interaction potentials using lattice element method, a methodology to calibrate and to validate the elasticity of solid constituents in heterogeneous porous media from experimentally measured nanoindentation moduli and imported scans from advanced imaging techniques is presented. Applied to computed tomography (CT) scans of two organic-rich shales, spatial variations of effective interaction potentials prove instrumental in capturing the effective elastic behavior of highly heterogeneous materials via the first two cumulants of experimentally measured distributions of nanoindentation moduli. After calibration and validation steps while implicitly accounting for mesoscale texture effects via CT scans, Biot poroelastic coefficients are simulated. Analysis of stress percolation suggests contrasting pathways for load transmission, a reflection of microtextural differences in the studied cases. This methodology to calibrate elastic energy content of real materials from advanced imaging techniques and experimental measurements paves the way to study other phenomena such as wave propagation and fracture while providing a platform to fine-tune effective behavior of materials given advancements in additive manufacturing and machine learning algorithms.
\end{abstract}

Keywords Effective poroelasticity $\cdot$ Heterogeneous $\cdot$ Mesoscale $\cdot$ Organic-rich shale

\section{Introduction}

It is well known that the effective mechanical and poromechanical properties of heterogeneous media depend on the chemical composition, mechanical properties of the constituents, as well as their spatial distributions. In recent years, the combination of multi-scale micromechanicsbased models and grid nanoindentation [15, 56], later coupled with wave-dispersive spectroscopy [16], has played a significant role in providing insights into the mechanics of heterogeneous porous solids. However, classical continuum micromechanics $[51,62]-$ and
Siavash Monfared

monfared@mit.edu

Hadrien Laubie

hlaubie@mit.edu

Farhang Radjai

fradjai@mit.edu

Mija Hubler

mija.hubler@colorado.edu

Roland Pellenq

pellenq@mit.edu

Franz-Josef Ulm

ulm@mit.edu
1 Department of Civil and Environmental Engineering, Massachusetts Institute of Technology, 77 Massachusetts Avenue, Cambridge, MA 02139, USA

$2\langle$ MSE $\rangle$ 2, UMI 3466 CNRS-MIT Energy Initiative, Massachusetts Institute of Technology, 77 Massachusetts Avenue, Cambridge, MA 02139, USA

3 LMGC, UMR-5508 CNRS, Universite de Montpellier, 163 Rue Auguste Broussonnet, 34090 Montpellier, France

4 Department of Civil, Environmental and Architectural Engineering, Colorado University, Boulder, CO 80309, USA

5 CINaM, CNRS, Aix Marseille Universite, Campus de Luminy, 13288 Marseille Cedex 09, France 
continuum microporomechanics [19, 20]-based homogenization methods, primarily built on Eshelby's inclusion problem [22] and scale separations, reduce the spatial distribution of the constituents and their mechanical interactions to effective textures, namely matrix/inclusion $[6,42]$ and granular $[13,26,27,32]$. The failure of continuum-based approaches to explicitly incorporate mesoscale texture effects by accounting for spatial distribution of constituents and the local variations of mechanical properties limits their ability to provide insights into the mechanics of highly heterogeneous media. In addition, perturbation-based solutions in statistical continuum mechanics are limited to small fluctuations in mechanical properties [7] and thus unable to capture heavy-tailed distributions characteristics of highly heterogeneous media. Moreover, challenges involved with defining a representative elementary volume that satisfies scale separability delineate the intrinsic limitations of the continuum model in dealing with highly heterogeneous media. Meanwhile, emerging modeling and simulation techniques propelled by high-performance computing has paved the way for multiscale, multi-physics material modeling, providing a link between microscale mechanisms and functional behavior at the macroscale. More recently, high-resolution imaging techniques such as computed tomography (CT) scans on porous heterogeneous solids (see, e.g., [28, 48]) have provided access to spatial distribution of pore and solid phases, paving the way to isolate mesoscale textural contribution to the effective elasticity via direct importation of such scans into a computational framework. Subsequently, the calibration of elastic energy content of a heterogeneous porous solid for a finite-sized simulation box against laboratory-measured mechanical data can serve as a departure point for simulating other phenomena such as wave propagation, plastic deformation, fracture. To address the limitations of continuum-based approaches in dealing with highly heterogeneous materials while utilizing advancements in high-performance computing and imaging techniques, we present a methodology to calibrate and to validate effective solid potentials of heterogeneous porous solids from experimentally measured nanoindentation data and imported CT scans. In addition to providing insights into the interplay of effective poroelastic behavior and microtexutral features, such a framework can be utilized to design new sustainable and durable materials with imposed effective mechanical behavior, with applications ranging from car manufacturing, aerospace industry to biological tissue engineering given the advancements in additive manufacturing and machine learning algorithms.

In this study, CT scans and laboratory-measured distributions of grid nanoindentation moduli on two organic-rich shales are employed. First two cumulants of experimentally measured distributions are used for calibration of effective interaction potentials of the solids. To this end, sub-volumes of characteristic length similar to that of a volume activated when probed by a nanoindenter are first extracted and grouped into calibration and validation structure sets. Utilizing lattice element method [3, 25, 54] and the framework of effective interaction potentials [35], elasticity of the solid phases, with spatial fluctuations within a phase, are calibrated using the calibration structure set. Then, the validation structure set is utilized as independent means for validation. With the elastic energy contents calibrated, Biot poroelastic coefficients are simulated using ensemble-based definitions for highly heterogeneous media [40]. Lastly, analyses of stress transmissions through larger sub-volumes extracted from the scans highlight the distinct underlying microtextural features and load-bearing phases in each case.

\section{Materials}

Computed tomography (CT) scans on samples from two different organic-rich shale formations (see [28] for details) are employed in this work. These CT scans are taken on samples of dimensions $64 \mu \mathrm{m} \times 64 \mu \mathrm{m} \times 100 \mu \mathrm{m}$ with a 5.4-keV energy source and a resolution of approximately $64 \mathrm{~nm}$. Such recently developed advanced imaging techniques provide the spatial distribution of different constituents of real materials. The same group of samples have been characterized extensively using instrumented nanoindentation, energy-dispersive X-ray spectroscopy (EDX) and X-ray diffraction (XRD) [1]. In addition, the organic contents have been modeled from reconstructed kerogen structures utilizing a hybrid reverse Monte-Carlo simulation (HRMC) method [12]. Herein, scan A refers to segmented CT data taken from Antrim formation with its organic content considered to be immature, while scan B represents the segmented CT data associated with the Haynesville formation with its organic content considered to be mature $[1,28]$. Maturity refers to the process of physical, chemical and structural evolution of organic content with geologic time due to exposure to high-pressure and high-temperature environments [47, 53]. Such evolution results in microtextural changes that impact the effective poroelastic behavior of organic-rich shales as a geocomposite [41]. Utilizing an informed Otsu's method, Hubler et al. [28] segmented the CT scans by grouping all their constituents into four phases-three solid phases (clay, inclusions, organics) and a pore phase. The organics phase is considered to be kerogen. The inclusions phase encompasses all inorganic solids other than clay. A cross section from scan B is displayed in Fig. 1, highlighting the well-preserved features of the raw data in the segmented image. 


\subsection{Calibration and validation structure sets}

Instrumented grid nanoindentation experiments have been shown to be an effective tool for characterizing the mechanical response of highly heterogeneous media $[15,56]$. Hence, the proposed methodology to calibrate effective elastic potential of constituents of a highly heterogeneous, multi-solid phase, porous medium utilizes instrumented nanoindentation data. This is done by extracting sub-volumes from the stack of CT scans, i.e., 3D volume, that correspond to the volume activated by a nanoindenter. Considering an average indentation depth of $900 \mathrm{~nm}$ results in an activated volume with a characteristic length of about $3 \times 900 \mathrm{~nm}$ [33]. This translates into cubic sub-volumes of $42 \times 42 \times 42$ voxels, given the $64 \mathrm{~nm}$ resolution of the CT scans. From each scan, 1000 spatially random sub-volumes are extracted. This forms the calibration structure sets (CSS). The remaining 1300 sub-volumes from scan A and 2886 sub-volumes from scan B make up the validation structure sets (VSS). The probability density function (PDF) of volume fractions for clay, inclusions, kerogen and pore phases $\left(f^{\text {clay }}, f^{\text {inc }}, f^{\text {ker }}, \phi\right)$ are plotted in Figs. 2 and 3 for scans A and B, respectively, and as a function different coarse-graining length scale, $\lambda$, which characterizes the length of the extracted cubic subvolumes and will be explored more later in this paper.

\section{Lattice element method and effective interaction potentials}

The lattice element method (LEM) $[3,25,54]$ is employed here to investigate both the elastic and the poroelastic behavior of highly heterogeneous real materials, utilizing the framework of effective interaction potentials [35] and ensemble-based definitions for Biot coefficients [40]. This is achieved by importing CT scans directly into LEM and discretizing the volume into a number of mass points.
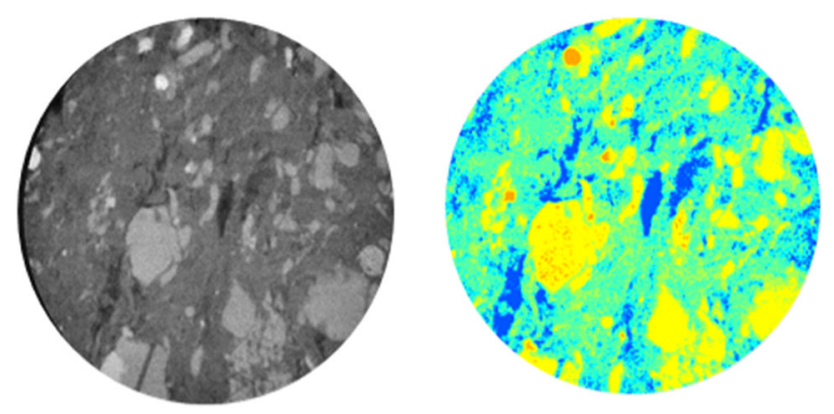

Fig. 1 A cross section of scan B displaying raw CT data (left) and the segmented image (right)

\subsection{Effective solid potentials}

The reference configuration (see Fig. 4) consists of $N_{\text {tot. }}=n_{x} \times n_{y} \times n_{z}$ total number of mass points arranged on a cubic lattice of unit size $a_{0}$, each exhibiting six degrees of freedom: three translations $\underline{\delta}$ and three rotations $\underline{\vartheta}$. Each mass point $i$ (reference position $\underline{x}_{i}$ and position in deformed configurations $\underline{X}_{i}$ ) interacts with a fixed number of neighboring points $j$ (a maximum of 26 corresponding to a cutoff radius $r_{\text {cut-off }}=\sqrt{3} a_{0}$ in Potential-of-Mean-Force (PMF) approaches) via a potential that considers both twobody and three-body interactions between two mass points $i$ and $j$, in the form:

$U_{i j}=U_{i j}^{s}+U_{i j}^{b} \quad \forall i \in V_{s}$

where $U_{i j}^{s}$ is a stretch term and $U_{i j}^{b}$ represent a bending term. For the case of linear poroelasticity applied to a structure close to its equilibrium position, this implies a harmonic expression for the two-body interaction term:

$U_{i j}^{s}=\frac{1}{2} \epsilon_{i j}^{n}\left(\frac{\delta_{j}^{n}-\delta_{i}^{n}}{l_{i j}^{0}}\right)^{2}$

with $\epsilon_{i j}^{n}$ denoting the axial energy parameter. Similarly, the three-body and rotational interactions read in the harmonic case [35]:

$$
\begin{aligned}
U_{i j}^{b}= & \frac{1}{2} \epsilon_{i j}^{t}\left\{\left(\frac{\delta_{j}^{b}-\delta_{i}^{b}}{l_{i j}^{0}}-\vartheta_{i}^{t}\right)^{2}+\left(\frac{\delta_{j}^{t}-\delta_{i}^{t}}{l_{i j}^{0}}+\vartheta_{i}^{b}\right)^{2}\right. \\
& +\left(\frac{\delta_{j}^{b}-\delta_{i}^{b}}{l_{i j}^{0}}-\vartheta_{i}^{t}\right)\left(\vartheta_{i}^{t}-\vartheta_{j}^{t}\right)+\left(\frac{\delta_{j}^{t}-\delta_{i}^{t}}{l_{i j}^{0}}+\vartheta_{i}^{b}\right) \\
& \left.\times\left(\vartheta_{j}^{b}-\vartheta_{i}^{b}\right)+\frac{1}{3}\left(\left(\vartheta_{j}^{b}-\vartheta_{i}^{b}\right)^{2}+\left(\vartheta_{i}^{t}-\vartheta_{j}^{t}\right)^{2}\right)\right\}
\end{aligned}
$$

where $\epsilon_{i j}^{t}$ is the transverse energy parameter and $l_{i j}^{0}=\left\|\underline{r}_{i j}\right\|$ (with $\underline{r}_{i j}=\underline{x}_{j}-\underline{x}_{i}=l_{i j}^{0} \underline{e}_{n}$ ) representing the distance between mass points $i$ and $j$ in the reference configuration. The energy parameters $\epsilon_{i j}^{(n, t)}$ of the solid can be calibrated to recover the desired effective elastic behavior for a homogeneous, isotropic or transversely isotropic, solid following the procedure outlined in [35]. The conjugated forces to translational degrees of freedom derive from the potential, $\underline{F}_{i}^{j}=-\partial U_{i j} / \partial \underline{\delta}_{i}$. For such a discrete system, the stresses are modeled using the virial expression [14]:

$\boldsymbol{\sigma}_{i}=\frac{1}{V_{i}} \sum_{j=1}^{N_{i}^{b}} \underline{r}_{i j} \otimes \underline{F}_{i}^{j}$ 


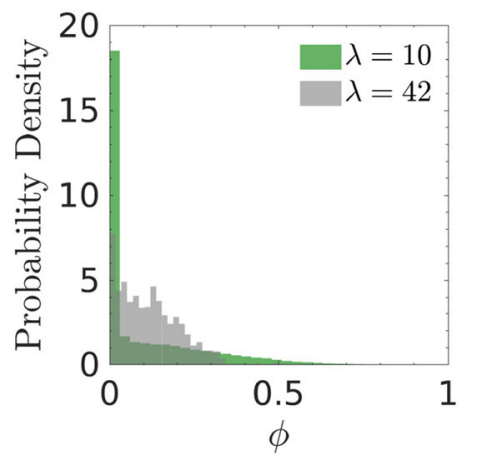

(a)

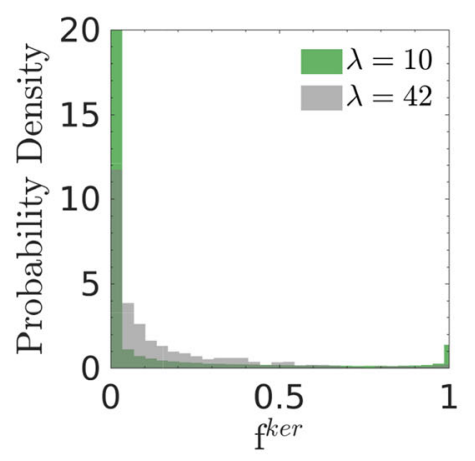

(d)

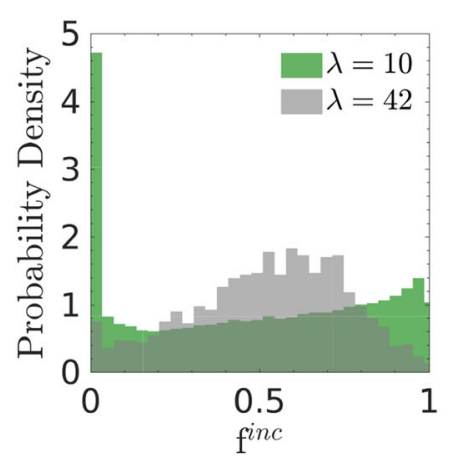

(g)

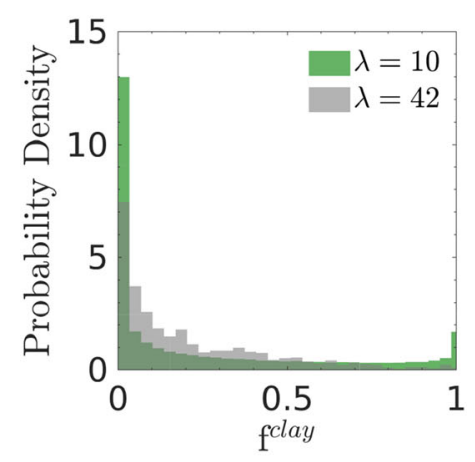

(j)

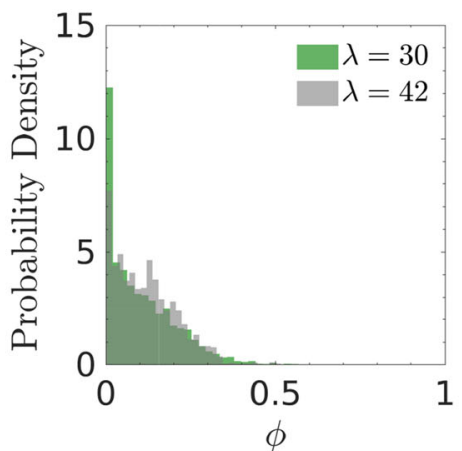

(b)

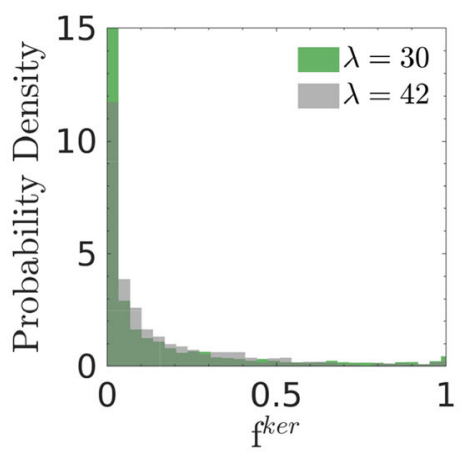

(e)

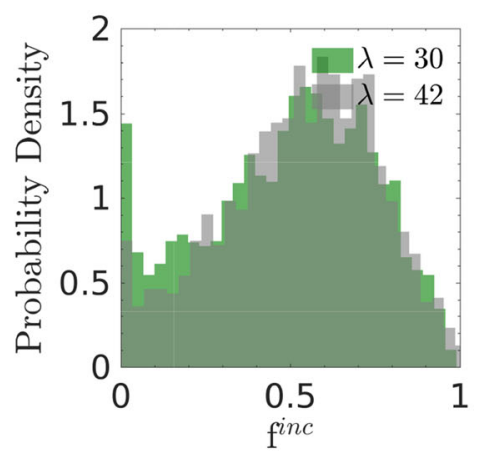

(h)

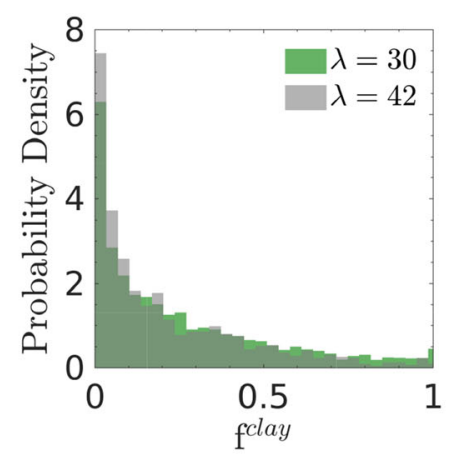

(k)

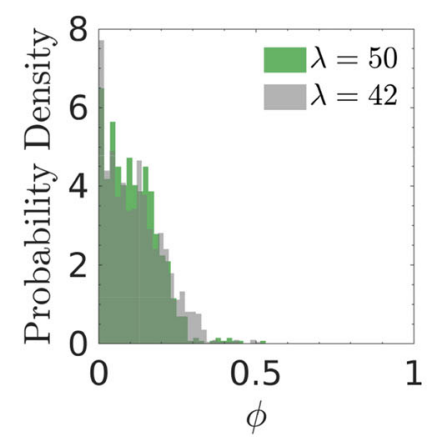

(c)

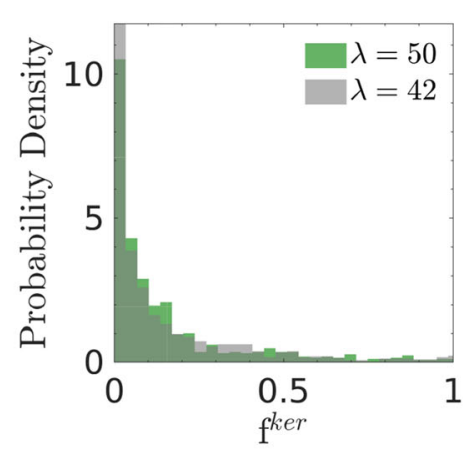

(f)

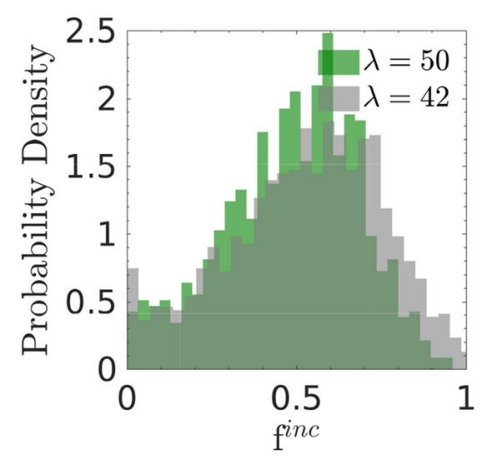

(i)

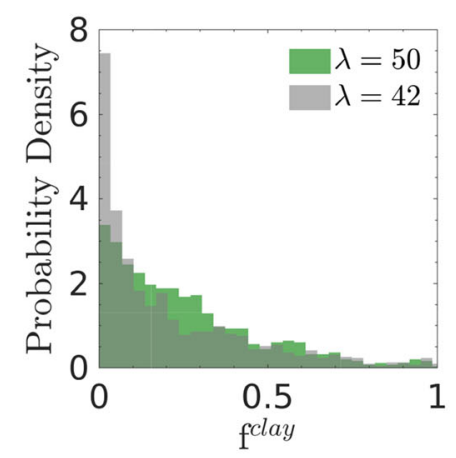

(I)

Fig. 2 Probability density functions for the volume fraction of the considered four phases-porosity (a-c), kerogen (d-f), inclusions $(\mathbf{g}-\mathbf{i})$ and clay $(\mathbf{j}-\mathbf{l})$ - as a function of coarse-graining length scale $\lambda$ in scan A 


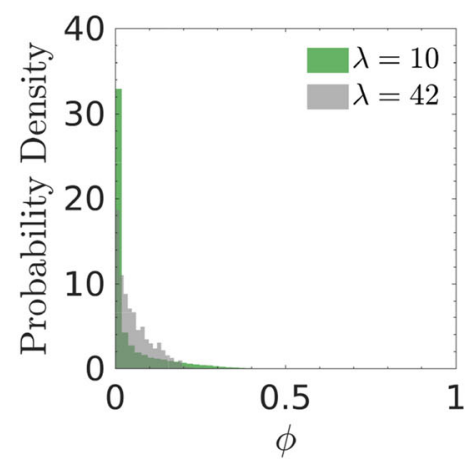

(a)

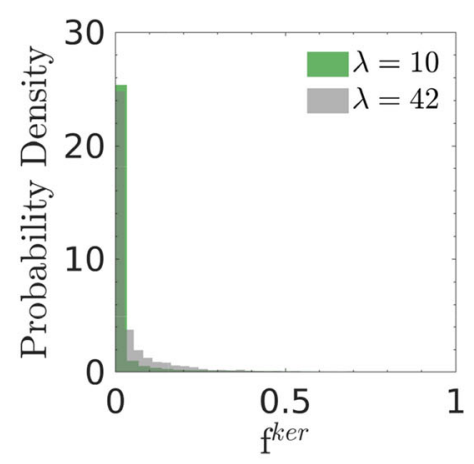

(d)

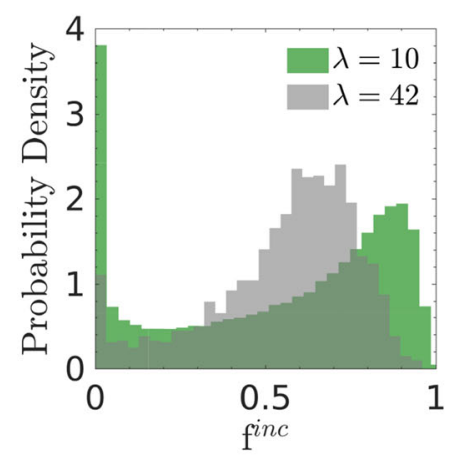

(g)

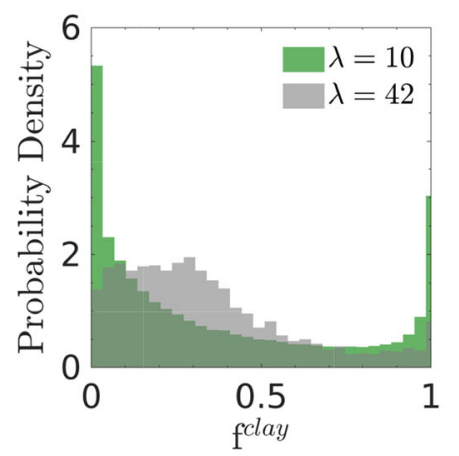

(j)

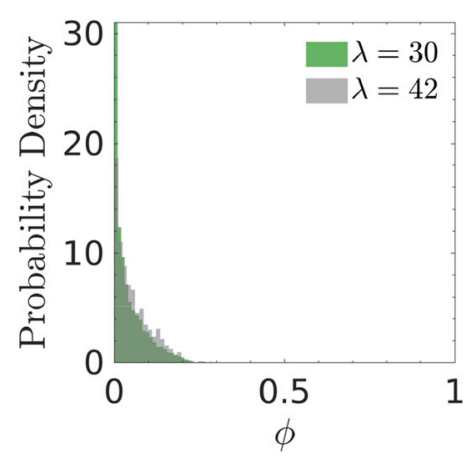

(b)

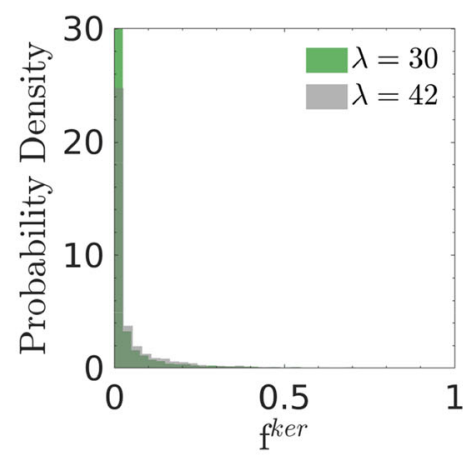

(e)

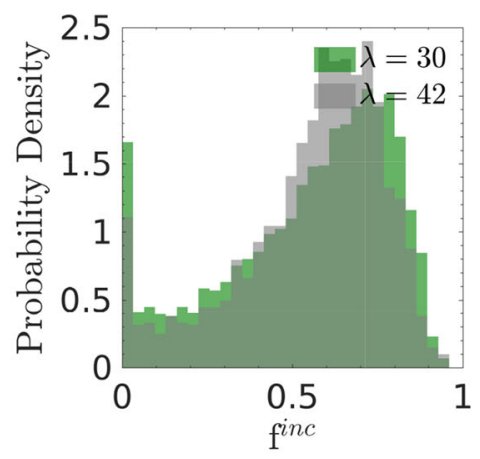

(h)

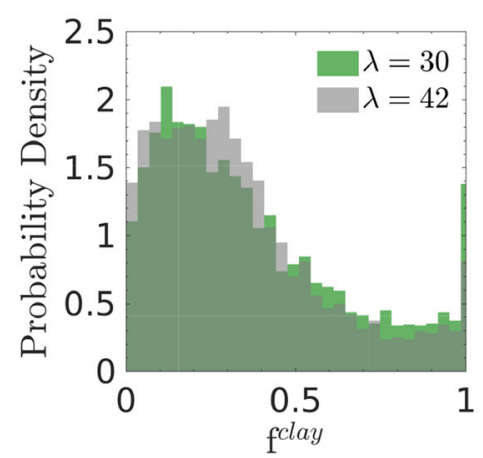

(k)

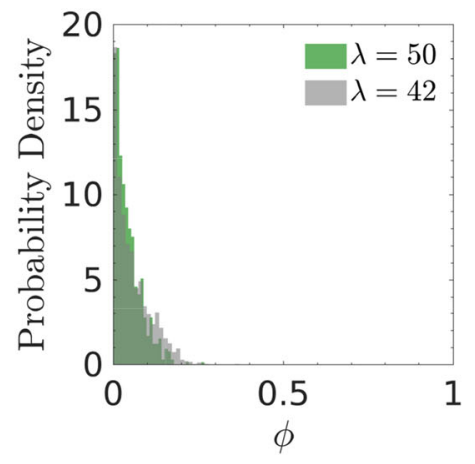

(c)

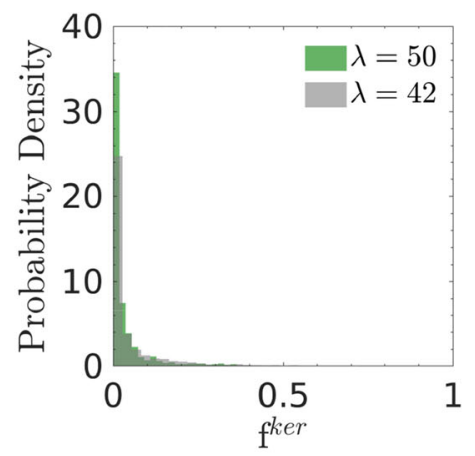

(f)

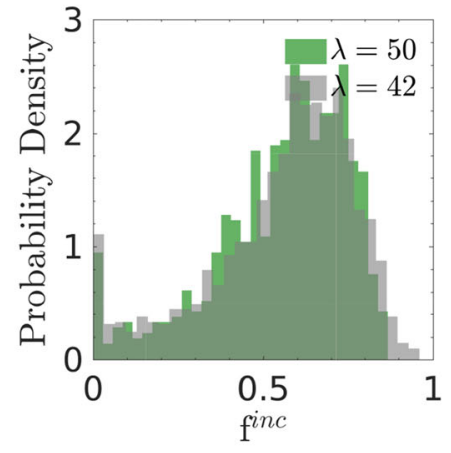

(i)

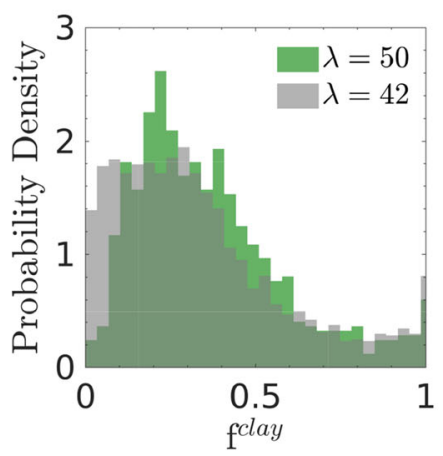

(I)

Fig. 3 Probability density functions for the volume fraction of the considered four phases-porosity $(\mathbf{a}-\mathbf{c})$, kerogen $(\mathbf{d}-\mathbf{f})$, inclusions $(\mathbf{g}-\mathbf{i})$ and clay (j-l) - as a function of coarse-graining length scale $\lambda$ in scan A 


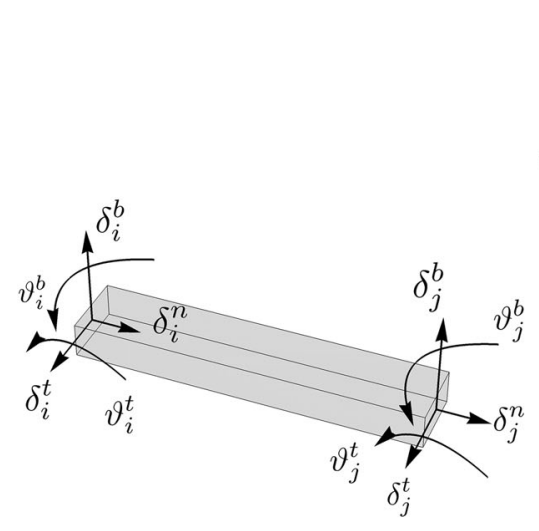

(a)

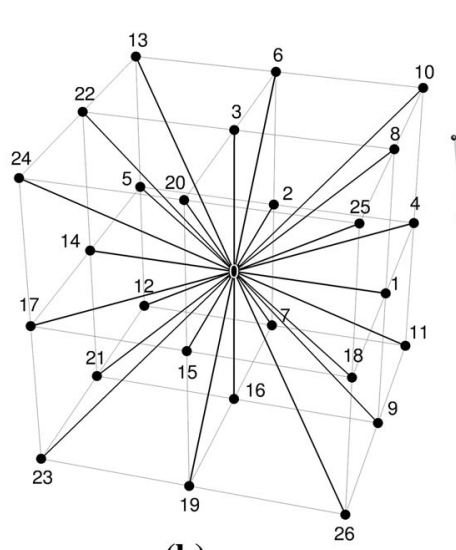

(b)

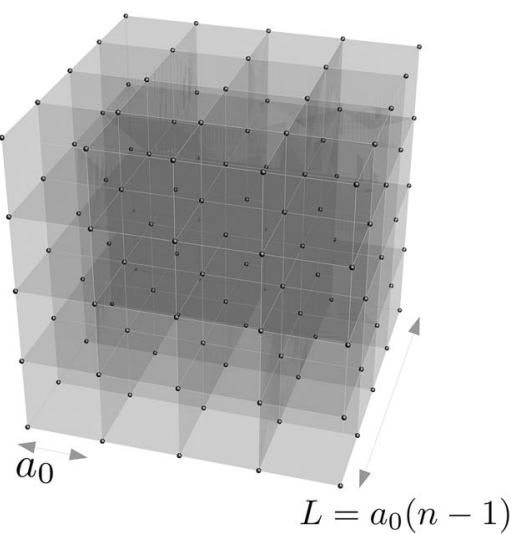

(c)

Fig. 4 a Degrees of freedom associated with a bond element between nodes $i$ and $j$; b D3Q26 unit cell; $\mathbf{c}$ simulation box

with $V_{i}=a_{0}^{3}$ denoting the volume of the unit cell and $N_{i}^{b}$ representing the number of node $i$ 's neighboring mass points. The virial expression provides a truly discrete description of the system as opposed to the continuumbased stress definition employed in classical finite-elementbased approaches. The stress in volume $V=$ $\left(n_{x}-1\right)\left(n_{y}-1\right)\left(n_{z}-1\right) V_{i}$ composed of a total of $N_{\text {tot. }}$ unit cells is simply the volume average of the local stresses; that is:

$$
\boldsymbol{\sigma}=\frac{1}{2 V} \sum_{i=1}^{N_{\text {tot. }}} V_{i} \boldsymbol{\sigma}_{i}
$$

what thus differs between different material domains is the interaction potential from which forces and moments are derived.

\subsection{Effective elasticity}

The state equations for stress, $\Sigma$ and porosity change, $\phi-\phi_{0}$, for linear poroelastic materials can be expressed as [11]:

$$
\begin{aligned}
& \Sigma=\frac{1}{V} \frac{\partial E_{\mathrm{pot}}}{\partial \mathbf{E}}=\mathbb{C}: \mathbf{E}-\mathbf{b} p \\
& \phi-\phi_{0}=-\frac{1}{V} \frac{\partial E_{\mathrm{pot}}}{\partial p}=\mathbf{b}: \mathbf{E}+\frac{p}{\mathrm{~N}}
\end{aligned}
$$

where $\mathbf{E}=\langle\boldsymbol{\varepsilon}\rangle_{V}$ is the average strain applied to the solidpore composite at the boundary $\partial V$, while pressure $p$ is imposed at the solid-pore interface. $\mathbb{C}$ is the fourth-order elastic stiffness tensor, $\mathbf{b}$ is the second-order tensor of Biot pore-pressure coefficients, and $\mathrm{N}$ denotes the solid Biot modulus. Given the objective of calibrating effective interaction potentials of the solid constituents from laboratory-measured indentation moduli, the effective elasticity of the porous composite needs to be determined first.
Following ensemble definitions of Monfared et al. [40], $\mathbb{C}$ can be obtained in the NVT-ensemble through imposing a regular displacement boundary condition $\underline{\xi}=\mathbf{E} \cdot \underline{x}$ at the boundary $(\partial V)$ of the simulation box while $p=0$. Given this mechanical boundary value problem, $(\mathbf{E}, p=0), \mathbb{C}$ is obtained by considering the curvature of the potential energy of the solid around the relaxed state:

$\mathbb{C}=\frac{1}{V} \frac{\partial}{\partial \mathbf{E}}\left(\frac{\partial E_{\text {pot }}^{s}}{\partial} \mathbf{E}\right)$

where $E_{\text {pot }}^{s}$ is the potential energy of the solid phase(s) which in this case coincides with the free energy of the solid phases in the defined NVT-ensemble.

\section{Methodology for calibrations of energy parameters}

Organic-rich shales are considered to exhibit a transversely isotropic elastic behavior at the length scale relevant to nanoindentation experiment. Laboratory-measured instrumented nanoindentation moduli, characterizing this anisotropic behavior, are employed as means for calibration of the effective potentials of the solid constituents. The experimental data represent a material's response to a nanoindenter as it probes the sample on a predetermined grid. For a transversely isotropic media, for which the nonzero components of the stiffness tensor-in Voigt notation-are $C_{11}=C_{22}, C_{12}, C_{13}=C_{23}, C_{33}, C_{44}=C_{55}$, while $C_{11}-C_{12}=2 C_{66}$; the indentation moduli can be expressed as [17]:

$$
M_{3}=2 \sqrt{\frac{C_{11} C_{33}-C_{13}^{2}}{C_{11}}\left(\frac{1}{C_{44}}+\frac{2}{\sqrt{C_{11} C_{33}}+C_{13}}\right)^{-1}}
$$


$M_{1} \approx \sqrt{\sqrt{\frac{C_{11}}{C_{33}}} \frac{C_{11}^{2}-C_{12}^{2}}{C_{11}} M_{3}}$

where $M_{1}$ and $M_{3}$ represent indentation moduli parallel to the plane of symmetry ( $\underline{e}_{1}$ and $\underline{e}_{2}$ directions) and axis of rotational symmetry ( $\underline{e}_{3}$-direction), respectively. The calibration procedure involves simulating the full stiffness tensor associated with each sub-volume in the calibration structure sets and utilizing Eqs. (9) and (10) to calculate the associated indentation moduli. Then, by the means of first two cumulants of the distributions of both simulated and experimental measured moduli, the effective potentials of the constituents are calibrated. The distributions of experimentally measured indentation moduli for scan A and scan B are plotted in Figs. 5 and 6, respectively. The first and second cumulants of a distribution, also known as the mean and the variance of a distribution, respectively, are defined as:

$$
\begin{aligned}
& \langle x\rangle_{c}=\langle x\rangle \\
& \left\langle x^{2}\right\rangle_{c}=\left\langle x^{2}\right\rangle-\langle x\rangle^{2}
\end{aligned}
$$

where $\langle x\rangle$ and $\left\langle x^{2}\right\rangle$ represent the first two moments of a distribution, respectively. In general, the $n$ th-moment of a distribution can be defined as:

$$
\left\langle x^{n}\right\rangle=\int p(x) x^{n} \mathrm{~d} x
$$

with $p(x)$ representing the probability density function (PDF) of random variable $x$. As previously discussed, the segmentation of CT scans reduce all the solid constituents to three solid phases, including an organic phase. Due to abundance of interfaces in these organic/inorganic porous media and in order to account for the interfaces (and discontinuities) not captured given the resolution of the imaging instrument, all voxels are modeled as springs in series, similar to colloidal models for cement $[37,38]$. For example in a simple onedimensional case, this implies that for voxels $i$ and $j$ in phase $a$, the effective spring constant $k_{\text {eff }}^{a}$ is defined as:

$\left.k_{\mathrm{eff}}^{a}=\frac{1}{k_{i}^{a}}+\frac{1}{k_{j}^{a}}\right)^{-1}$

For simulations in LEM, the elasticity of each solid constituents is calibrated through the framework of effective interaction potentials [35], given a finite-sized simulation box. At the interface of different phases, the mechanical interaction for node $i$ in phase $a$ and neighboring node $j$ in phase $b$ (and vice-versa) is modeled as follows:

$$
\left.{ }^{\text {int. }} \epsilon_{i j}^{(n, t)}={ }^{\text {int. }} \epsilon_{j i}^{(n, t)}=2 \frac{1}{\text { bulk } \epsilon_{i j}^{(n, t)}}+\frac{1}{\text { bulk } \epsilon_{j i}^{(n, t)}}\right)^{-1}
$$

where ${ }^{\text {bulk }} \epsilon_{i j}^{(n, t)}$ and bulk $\epsilon_{j i}^{(n, t)}$ denote the potential parameters calibrated to produce the desired elasticity of phases $a$ and $b$, respectively, while ${ }^{\text {int. }} \epsilon_{i j}^{(n, t)}={ }^{\text {int. }} \epsilon_{j i}^{(n, t)}$ quantify mechanical interaction at the interface of the two phases. The sensitivity of the results to this definition for the interfaces will be discussed later.

\subsection{Input elasticity of the organic phase}

The organic phase is considered to be kerogen and to exhibit isotropic elastic behavior, fully characterized by two elastic moduli. Based on the molecular simulations of Bousige et al. [12] on reconstructed organic structures and multi-scale molecular informed micromechanics model of Monfared and Ulm [41], $v_{\text {ker. }}^{A}=0.25$ and $M_{\text {ker. }}^{A}=$ 10.27 GPa for scan A and $v_{\text {ker. }}^{B}=0.25$ and $M_{\text {ker. }}^{B}=$ $2.24 \mathrm{GPa}$ for scan B are chosen as inputs. $M$ refers to isotropic indentation modulus, defined as $M=E /\left(1-v^{2}\right)$, $v$ denotes Poisson's ratio and $E$ represents Young's modulus. The distinct elasticity of the kerogen phase for these two samples is a consequence of their maturity level and their initial compositions.

\subsection{Degrees of freedom: clay and inclusion effective potentials}

Since the CT data are oblivious to pore space below $64 \mathrm{~nm}$, resolution of the CT data per voxel, and represent a length scale too coarse to account for the variations of clay mineral platelets and their orientations (see, e.g., [21]), the clay phase is modeled as a porous aggregate of clay particles effectively exhibiting a transversely isotropic elastic behavior. To fully capture this behavior, five elastic constants are needed. To this end, the values obtained through the inversion of ultrasonic pulse velocity data through the multi-scale molecular informed micromechanics model of Monfared and Ulm [41] are considered, i.e., $C_{11}=103.0 \mathrm{GPa}, C_{12}=41.6 \mathrm{GPa}, C_{13}=34.1 \mathrm{GPa}, C_{33}=$ $43.3 \mathrm{GPa}$ and $C_{44}=7.7 \mathrm{GPa}$. However, such transversely isotropic behavior cannot be reproduced in LEM, in its current formulation, since it violates [35]:

$$
C_{12} \leq C_{66}\left(\text { i.e. } \quad C_{12} \leq \frac{1}{3} C_{11}\right) ; C_{13} \leq C_{44}
$$

Thus, clay is modeled as a quasi-transversely isotropic material in a reduced stiffness space. Specifically, only four elastic moduli, instead of five, are used for calibration of $\epsilon_{i j}^{(n, t)}$ for the clay phase. The four elastic moduli are $M_{1}$ and $M_{3}$ as defined in Eqs. (9) and (10), respectively; as well as Voigt-Reuss-Hill averages for bulk, $K_{\mathrm{VRH}}$, and shear, $G_{\mathrm{VRH}}$, moduli [9] for a material exhibiting a hexagonal elastic symmetry which includes the transversely isotropic case. Thus, the following elastic constants describe the quasi-transversely isotropic behavior of the clay phase in 


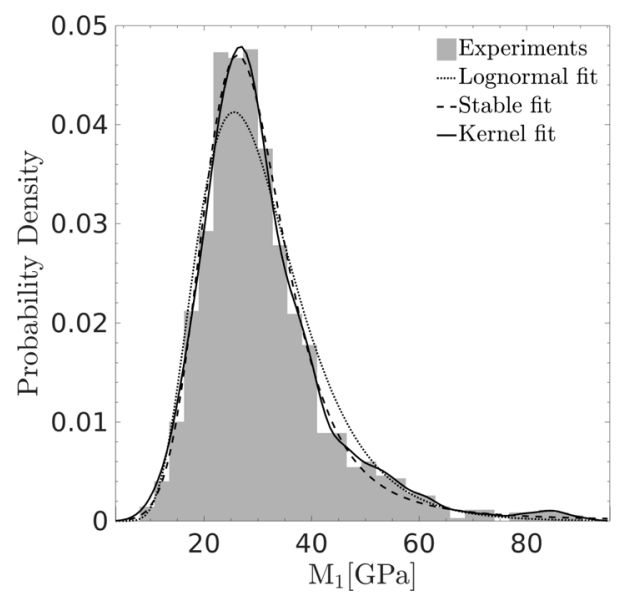

(a)

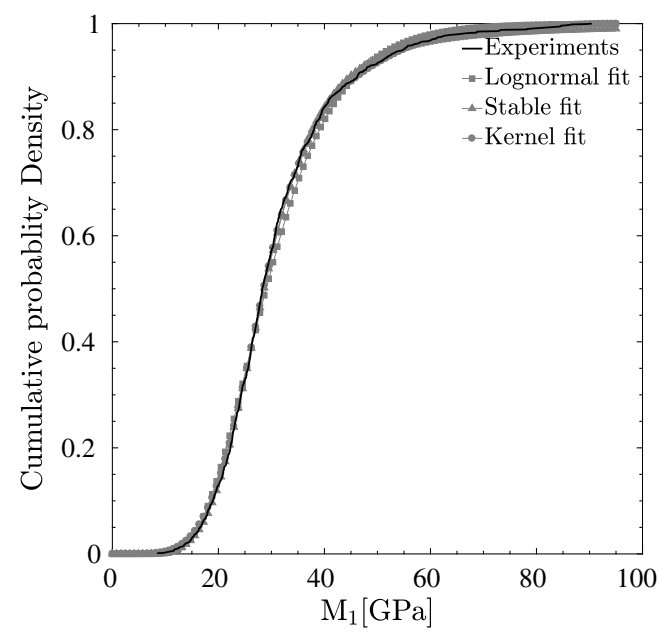

(c)

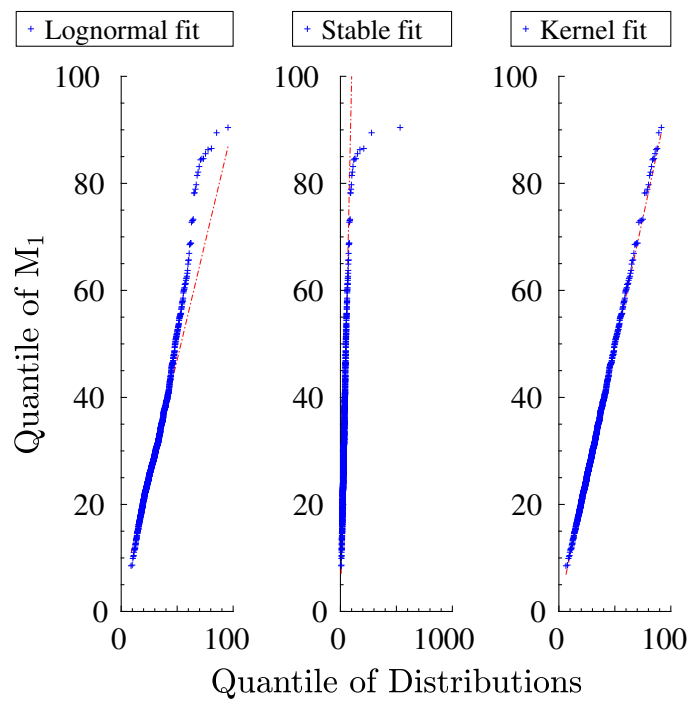

(e)

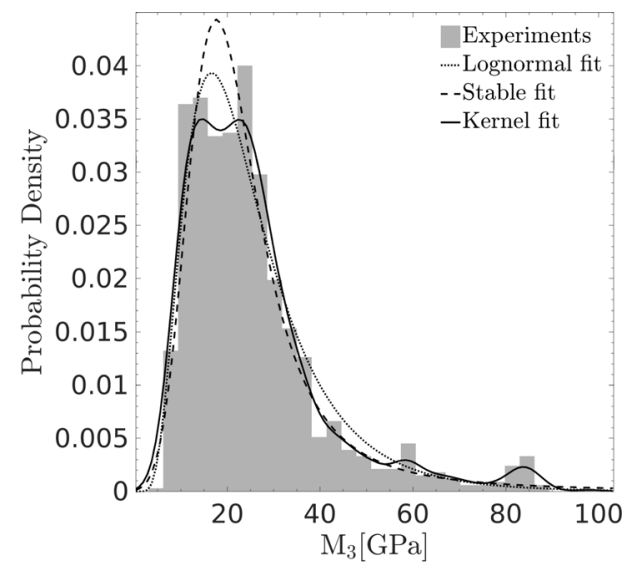

(b)

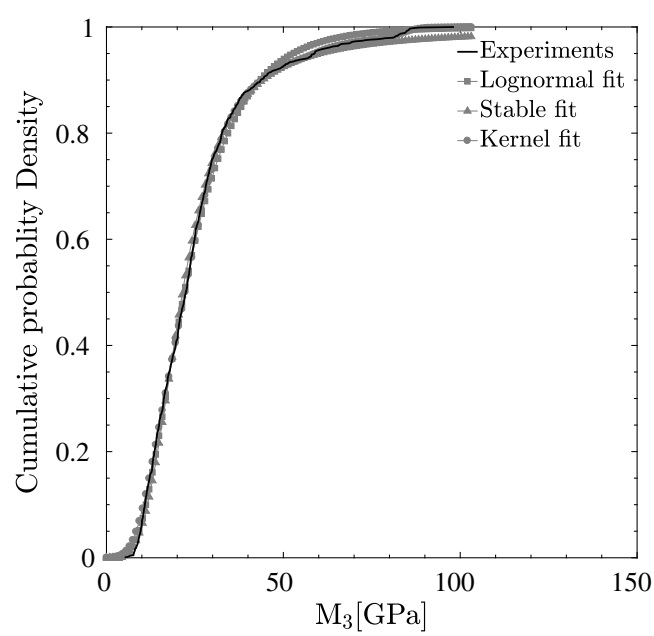

(d)

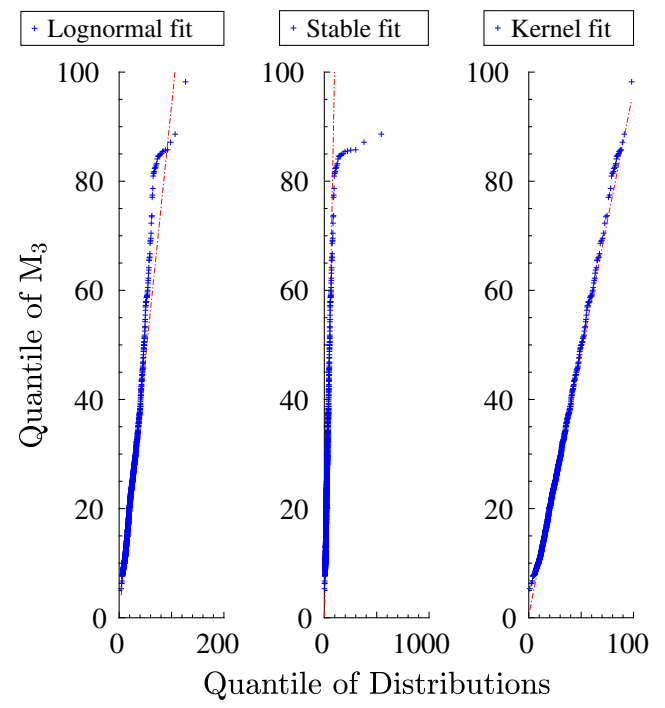

(f)

Fig. 5 Probability density (a, b), cumulative probability density (c, d) and quantile-quantile plots (e, f) for experimentally measured nanoindentation moduli for scan A with fitted lognormal, Stable and nonparametric normal kernel distributions 
this work: $\quad M_{1}=62.35 \mathrm{GPa}, \quad M_{3}=29.25 \mathrm{GPa}$, $K_{\mathrm{VRH}}=46.81 \mathrm{GPa}$, and $G_{\mathrm{VRH}}=17.63 \mathrm{GPa}$. Since voxels belonging to each phase are modeled as springs in series, the calibration of energy parameters is based on $\left(M_{1}^{*}, M_{3}^{*}, G^{*}, K^{*}\right)$ where $M_{1}^{*}=M_{1} / 2, M_{3}^{*}=M_{3} / 2, K^{*}=$ $E^{*} /(3(1-2 v))$ and $G^{*}=E^{*} /(2(1+v))$ where $E^{*}=$ $0.5\left(M_{1}^{*}+M_{3}^{*}\right)(1-v) \quad$ and $\quad v=\left(3 K_{\mathrm{VRH}}-2 G_{\mathrm{VRH}}\right) /$ $\left(6 K_{\mathrm{VRH}}+2 G_{\mathrm{VRH}}\right)$. It is well known that clay exhibits a range of elastic behaviors based on its type [4, 24, 31, 31, 49, 57]. Additionally, experimental observations of [10] using transmission electron microscopy coupled with energy-dispersive X-ray spectroscopy (TEMEDX) hint at the spatial heterogeneity of clay particles even at nanometer length scales. To capture this, small fluctuations around the mean values of $\epsilon_{i j}^{(n, t)}$ in the clay phase are introduced for all 1000 sub-volumes during calibration. Specifically, for the links in the plane of symmetry $\underline{e}_{1} \times \underline{e}_{2}\left[\left(\epsilon_{1}^{n}, \epsilon_{1}^{t}\right)\right.$ for the 4 box-links of rest length $l^{0}=a_{0}$ oriented in the $\underline{e}_{1}$ - and $\underline{e}_{2}$-directions, and $\left(\epsilon_{4}^{n}, \epsilon_{4}^{t}\right)$ for the 4 in-plane diagonals of length $\sqrt{2} a_{0}$; see Fig. 4 ] fluctuations around a mean value are modeled as a Gaussian distribution:

$\epsilon_{i}^{(n, t)} \sim N\left(\bar{\epsilon}_{i}^{(n, t)}, S_{1}^{2}\right) \quad \forall i \in\{1,4\}$

where $\bar{\epsilon}_{i}^{(n, t)}$ denotes the average value, calibrated based on the effective potential framework of Laubie et al. [35] and $S_{1}^{2}$ represents the directionally dependent variance, i.e., in $\underline{e}_{1}$, introduced as a degree of freedom to quantify spatial fluctuations in clay elasticity. Additionally, for the 2 boxlinks oriented in the $\underline{e}_{3}$-direction, i.e., $\epsilon_{3}^{n}$, spatial fluctuations are introduced similar to Eq. (17):

$\epsilon_{3}^{n} \sim N\left(\bar{\epsilon}_{3}^{n}, S_{3}^{2}\right)$

with $S_{3}^{2}$ representing the directionally dependent variance in $\underline{e}_{3}$ while assuming distributions outlined in Eqs. (17) and (18) are independently distributed. In summary, two degrees of freedom, $S_{1}^{2}$ and $S_{3}^{2}$ are introduced to quantify the spatial heterogeneity of clay elasticity. Furthermore, the inclusions phase encompasses a variety of minerals with a range of elasticity. Figure 7 displays pie-charts for the components of what is considered to be the inclusions phase based on XRD data [41]. Similar to the clay phase, the inclusions phase is modeled as a porous (sub-CT resolution) aggregate of polycrystals effectively exhibiting an isotropic elastic behavior. In order to capture the range of elasticity represented by this diverse group of minerals while maintaining the degrees of freedom at a minimum, $\epsilon_{i j}^{(n, t)}$ for the inclusions phase can be written as: $\epsilon_{i j}^{(n, t)}=k_{i j}^{(n, t)}\left(l_{i j}^{0}\right)^{2}=k \Gamma_{i j}^{(n, t)}\left(l_{i j}^{0}\right)^{2}$

where $k$ is a directionally independent force constant and $\Gamma_{i j}^{(n, t)}$ is a directionally dependent force constant pre-factor. Since $\epsilon_{i j}^{(n, t)} \geq 0$ for instability reasons, a two-parameter univariate Weibull distribution $[59,60]$ characterized by a shape factor, $\alpha \in \mathbb{R}_{>0}$, and a scale factor, $\beta \in \mathbb{R}>0$, is chosen to model the distribution of force constant $k$ for subvolume $m$ :

${ }^{m} k \sim W\left(\alpha_{m}, \beta_{m}\right)$

As an example, Fig. 8 illustrates the spatial distribution of $\epsilon_{1}^{n}$ in a cross section of one of the sub-volumes. In summary, each sub-volume used for calibration has two degrees of freedom associated with the inclusions phase, while $S_{1}^{2}$ and $S_{3}^{2}$ degrees of freedom belong to the whole set of sub-volumes. Hence, with a calibration structure set of 1000 sub-volumes, there are $1000 \times 2+2=2002$ total degrees of freedom for each scan. For initial values and based on Fig. 7, scan A seems to be dominated by Quartz. Thus, as initial guesses for its isotropic elasticity, $\mathrm{K}_{\mathrm{inc}}^{\mathrm{A}}=$ 37.90 $\mathrm{GPa}$ and $\mathrm{G}_{\text {inc }}^{\mathrm{A}}=44.30 \mathrm{GPa}$ are chosen [39]. XRD data, as shown in Fig 7, suggest that the inclusions phase for scan B is dominated by Quartz and Calcite. As an initial guess, a Voigt-Reuss-Hill (VRH) average assuming each phase contributes with equal weights is employed. This translates into $\mathrm{K}_{\mathrm{inc}}^{\mathrm{B}}=46.98 \mathrm{GPa}$ and shear modulus $\mathrm{G}_{\mathrm{inc}}^{\mathrm{B}}=$ $35.42 \mathrm{GPa}$ using elastic properties as reported in [39].

\subsection{Numerical optimization}

The optimization was performed using NLOPT library [29] with Constrained Optimization By Linear Approximation (COBYLA) algorithm [44, 45] while utilizing message passing interface (MPI) to simulate 1000 sub-volumes at a time for calibration of each scan and for each iteration step of the optimization process. The objective function is defined as:

$$
\min _{d}(1-r)
$$

where $r$, the correlation coefficient reads:

$$
r=\frac{\sum_{i}^{n}\left(x_{i}-\langle x\rangle\right)\left(y_{i}-\langle y\rangle\right)}{\sqrt{\sum_{i}^{n}\left(x_{i}-\langle x\rangle\right)^{2} \sum_{i}^{n}\left(y_{i}-\langle y\rangle\right)^{2}}}
$$

where

$$
\begin{aligned}
& x=\left\{\left\langle{ }^{\text {sim. }} M_{1}\right\rangle_{c},\left\langle{ }^{\text {sim. }} M_{1}^{2}\right\rangle_{c}^{0.5},\left\langle{ }^{\text {sim. }} M_{3}\right\rangle_{c},\left\langle{ }^{\text {sim. }} M_{3}^{2}\right\rangle_{c}^{0.5}\right\} \\
& y=\left\{\left\langle{ }^{\text {exp. }} M_{1}\right\rangle_{c},\left\langle{ }^{\text {exp. }} M_{1}^{2}\right\rangle_{c}^{0.5},\left\langle{ }^{\text {exp. }} M_{3}\right\rangle_{c},\left\langle{ }^{\text {exp. }} M_{3}^{2}\right\rangle_{c}^{0.5}\right\}
\end{aligned}
$$




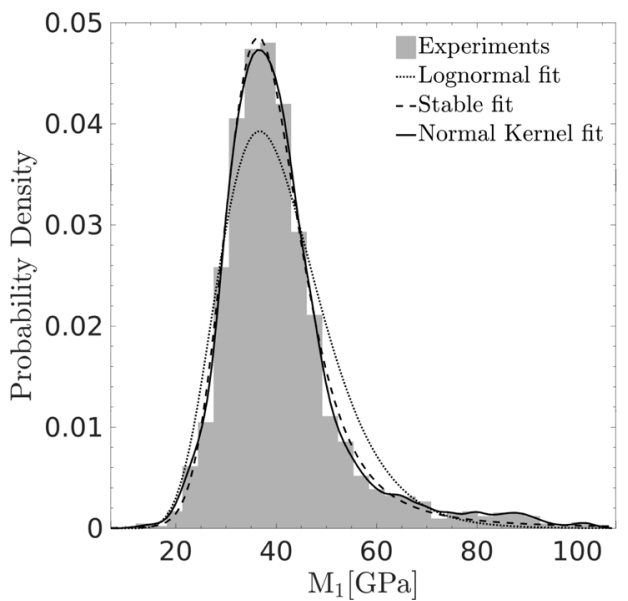

(a)

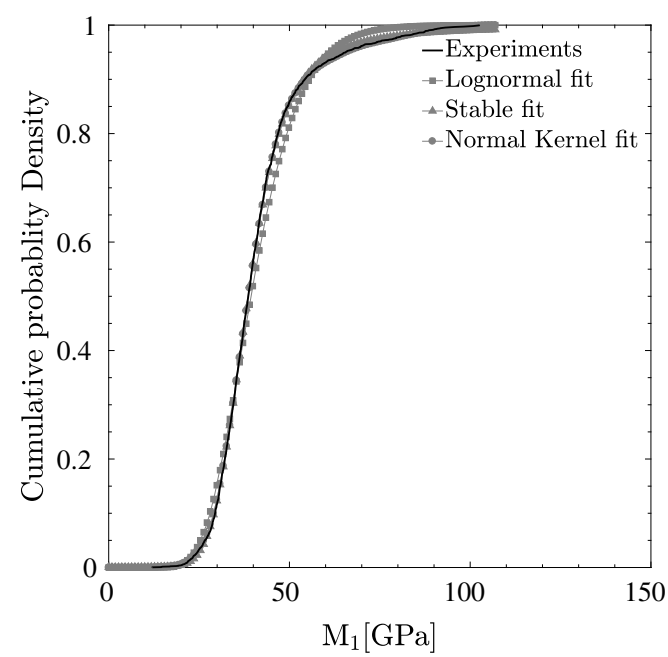

(c)

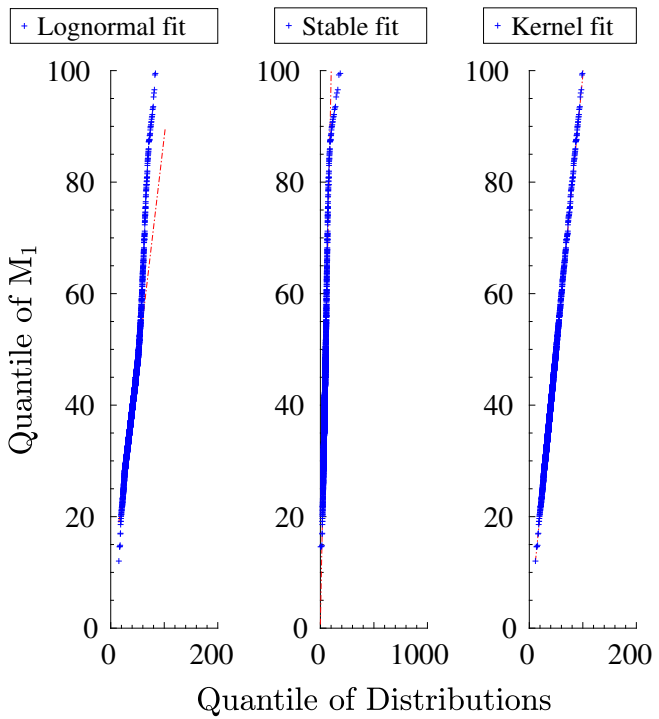

(e)

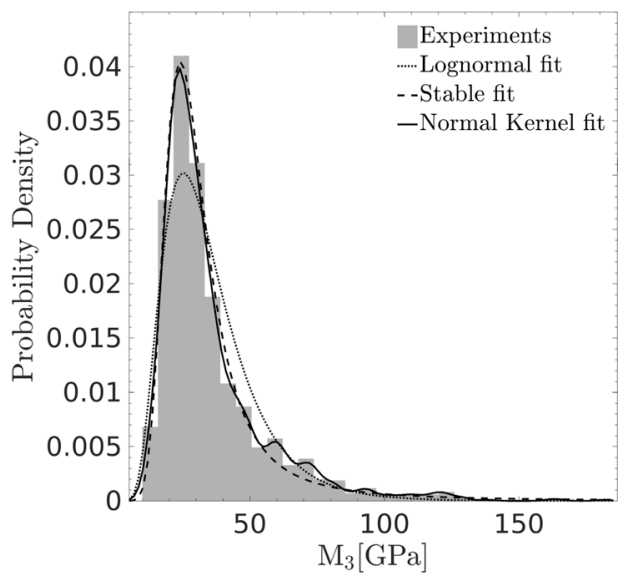

(b)

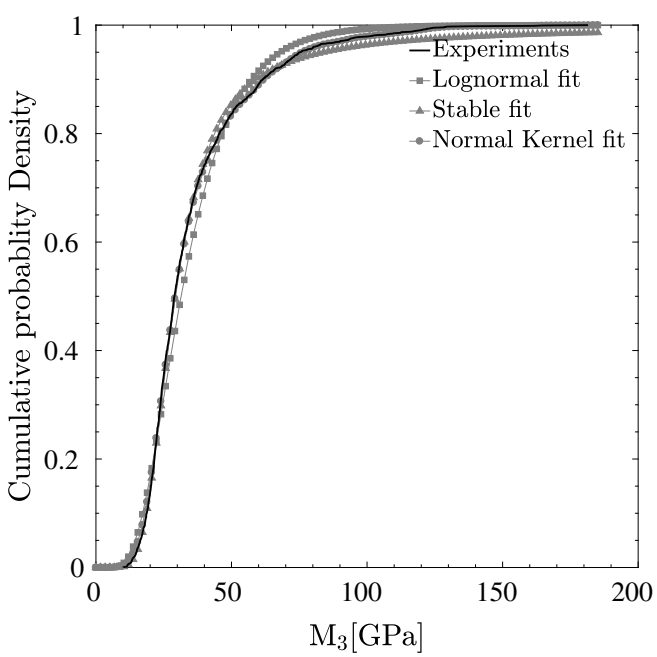

(d)

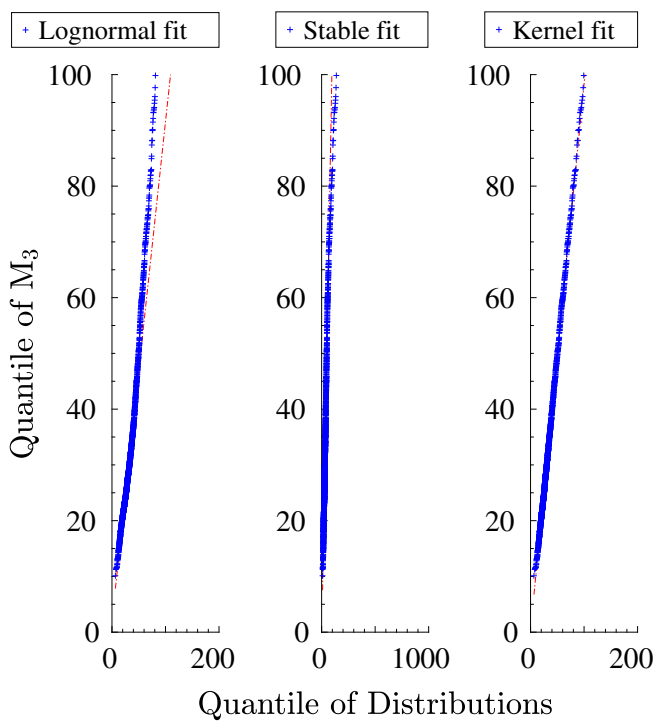

(f) 
4Fig. 6 Probability density (a, b), cumulative probability density (c, d) and quantile-quantile plots $(\mathbf{e}, \mathbf{f})$ for experimentally measured nanoindentation moduli for scan B with fitted lognormal, Stable and nonparametric normal kernel distributions

with $d$ denoting the set containing degrees of freedom, $d=\left\{S_{1}^{2}, S_{3}^{2},\left\{\alpha_{i}\right\},\left\{\beta_{i}\right\}\right\}$ with $|d|=2002$ given 1000 subvolumes from each scan employed for calibration.

\section{Results}

\subsection{Calibration and validation results}

The first two cumulants of the distributions for calibrated indentation moduli for both scans and the corresponding

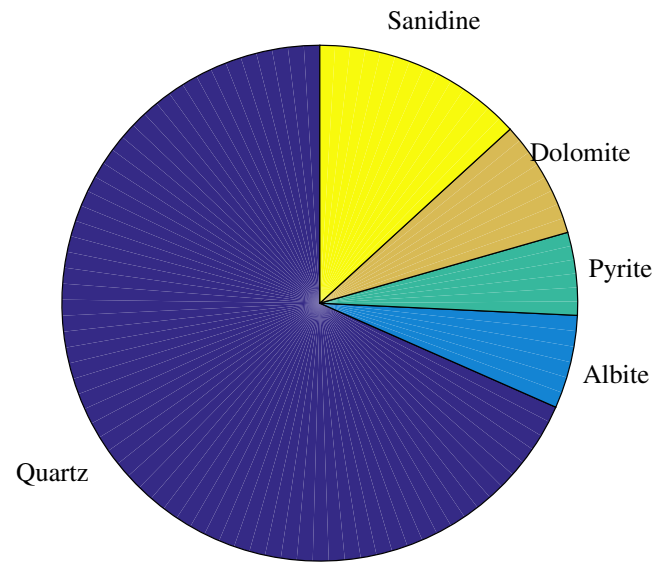

(a) cumulants based on experimentally measured distributions are summarized in Table 1. In addition, these values are plotted in Fig. 9 as a representation of the calibration quality with $r=0.991$ for scan A and $r=0.994$ for scan B. The probability density and cumulative probability density functions for the optimized shape factors, $\left\{\alpha_{i}\right\}$, and scale factors $\left\{\beta_{i}\right\}$ for both scans $\mathrm{A}$ and $\mathrm{B}$ are displayed in Fig. 10. These parameters characterize the Weibull distributions for the energy parameters of the inclusions phase. Furthermore, for the clay phase in scan $\mathrm{A},{ }^{A} S_{1}^{2}=$ $52.91 \mathrm{GPa}^{2}$ and ${ }^{A} S_{3}^{2}=50.48 \mathrm{GPa}^{2}$ and for scan $\mathrm{B},{ }^{B} S_{1}^{2}=$ $15.16 \mathrm{GPa}^{2}$ and ${ }^{B} S_{3}^{2}=15.79 \mathrm{GPa}^{2}$ were obtained. In terms of elastic moduli, Fig. 11 displays the distributions for inclusions elasticity for both scans, while the three-component Gaussian mixture fit parameters for both cases are summarized in Table 2. Similarly, the small variations in

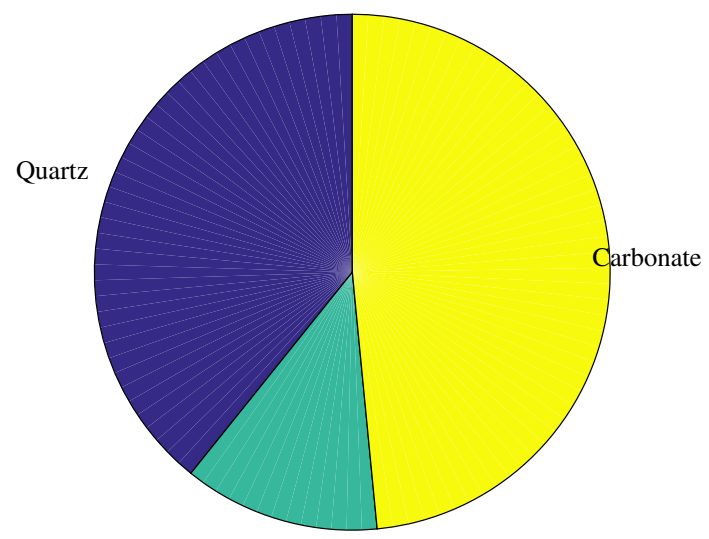

Feldspar

(b)

Fig. 7 Mineralogical composition of the inclusions phase from XRD data as reported in [41] for scan A (a) and scan B (b)

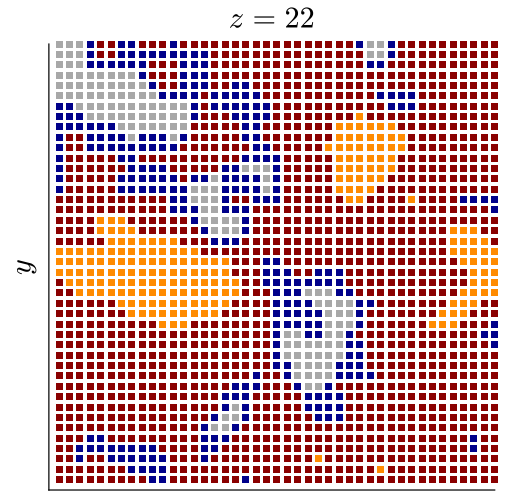

(a)

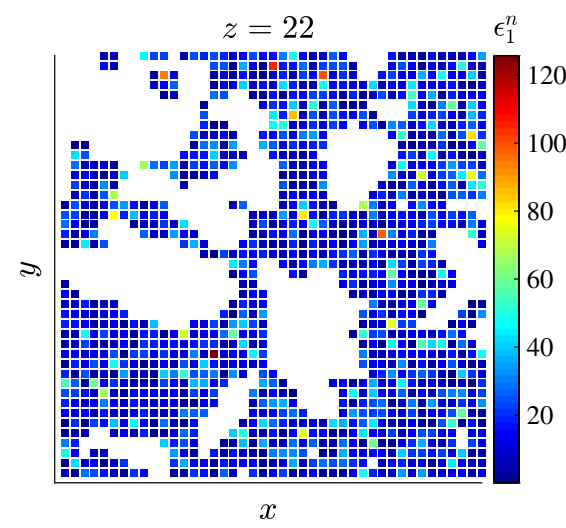

(b)

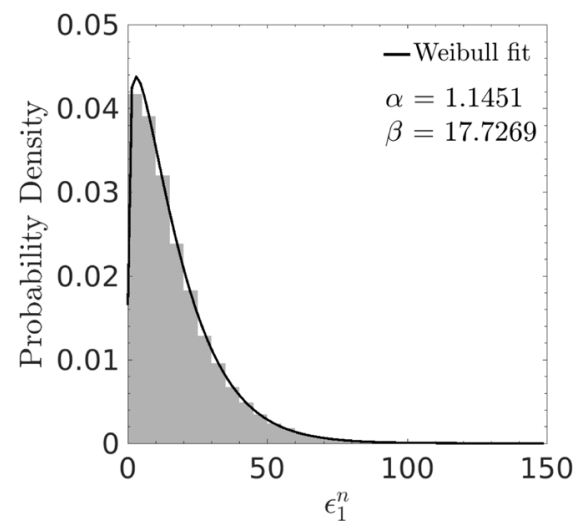

(c)

Fig. 8 a Cross section of a discretized sub-volume extracted from CT scans. Colors red, orange, blue and gray correspond to inclusions, clay, pore and kerogen phases, respectively.; b Color map for $\epsilon_{1}^{n}$ variations in the inclusions phase as shown in a; $\mathbf{c}$ Weibull distribution of $\epsilon_{1}^{n}$ energy parameter, as an example, shown spatially in $\mathbf{b}$ (color figure online) 
Table 1 First and second cumulants of the distributions of indentation moduli of calibration structure set and experimentally measured

\begin{tabular}{lllllllll}
\hline & $\left\langle{ }^{\text {exp. }} M_{1}\right\rangle_{c}$ & $\left\langle{ }^{\text {exp. }} M_{1}^{2}\right\rangle_{c}$ & $\left\langle{ }^{\text {exp }} M_{3}\right\rangle_{c}$ & $\left\langle{ }^{\text {exp. }} M_{3}^{2}\right\rangle_{c}$ & $\left\langle{ }^{\text {sim. }} M_{1}\right\rangle_{c}$ & $\left\langle{ }^{\text {sim. }} M_{1}^{2}\right\rangle_{c}$ & $\left\langle\text { sim. } M_{3}\right\rangle_{c}$ & $\left\langle{ }^{\text {sim. }} \cdot M_{3}^{2}\right\rangle_{c}$ \\
\hline Scan A & 30.933 & 144.816 & 25.483 & 235.540 & 29.271 & 197.354 & 36.020 \\
Scan B & 40.892 & 151.057 & 35.528 & 426.536 & 40.923 & 175.110 & 35.167 \\
\hline
\end{tabular}

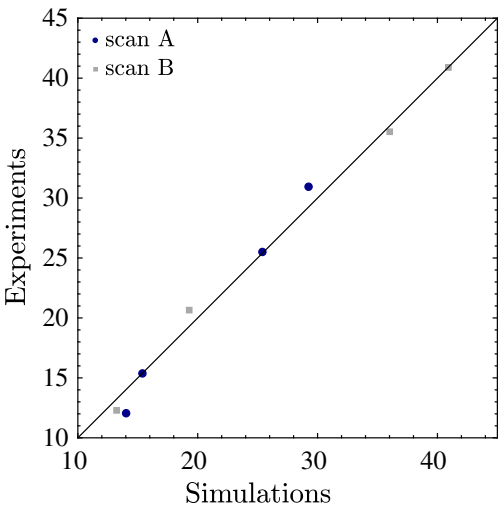

Fig. 9 Optimized and experimentally measured $\left\langle M_{1}\right\rangle_{c},\left\langle M_{1}^{2}\right\rangle_{c}^{0.5},\left\langle M_{3}\right\rangle_{c}$ and $\left\langle M_{3}^{2}\right\rangle_{c}^{0.5}$ for scans $\mathrm{A}$ and $\mathrm{B}$

the elasticity of clay phase are shown in Fig. 12. To validate the results, the VSS is employed for each scan. The obtained distributions for $\left\{\alpha_{i}\right\}$ and $\left\{\beta_{i}\right\}$ (see Fig. 10) are employed to generate random numbers in a forward applications for simulating sub-volumes belonging to VSS of each scan. The resulting distributions are plotted in Fig 13 for scan A and Fig 14 for scan B and summarized in Table 3 in terms of first two cumulants. This provides independent means to validate the calibrated effective interaction potentials.

\subsection{Biot poroelastic coefficients}

Ensemble-based definitions for Biot poroelastic coefficients [40] are employed to simulate poroelastic response of these highly heterogeneous, porous solids given the calibrated effective interaction potentials of its solid constituents. To model the effect of pressure in the pore domain on the deformation behavior of the solid phase(s), the saturated drained case is considered. This case implies a hydrostatic stress state, $\boldsymbol{\sigma}=-p \mathbf{1}$, which can be simulated in the pore domain by imposing a central force $\left(\underline{F}_{i}^{j}=F_{i}^{j, n} \underline{e}_{n}\right)$ on each link associated with a node inside the pore domain through the following relationship [40]:

$F_{i}^{j, n}=-\frac{6 p}{r_{i j}} \frac{V_{p}}{n_{\ell}^{p}}$

where $V_{p}$ represents the volume of the pore domain and $n_{\ell}^{p}$ denotes the number of links associated with nodes
Fig. 10 Probability density and cumulative probability density functions for optimized shape factors, $\left\{\alpha_{i}\right\}$, and scale factors, $\left\{\beta_{i}\right\}$, characterizing the Weibull distributions for the inclusions phase for scan A (a, c, e, g) and scan B (b, d, f, h)

belonging to the pore space. Equation (25) defines the interaction between pore and solid mass points in the form of externally supplied work. This perturbation of the system's equilibrium is resolved through the theory of minimum potential energy as a new equilibrium position is sought through energy minimization $[35,40]$.

The second-order tensor of Biot pore-pressure coefficients, $\mathbf{b}$, is determined in the $\mu \mathrm{VT}$-ensemble at the composite scale [solid(s) + pore] where the macroscopic strain $\mathbf{E}$ is zero while a constant pressure $p$ prevails in the pore space, exerting this pressure onto the solid-pore interface. For such a boundary condition, $(\mathbf{E}=\mathbf{0}, p), \mathbf{b}$ can be expressed in terms of equilibrated interaction forces [40]:

$\mathbf{b}=-\frac{\Sigma^{(\mu \mathrm{VT})}}{p}=\frac{1}{p}\left(\frac{1}{2 V} \sum_{i \in V_{s}} \sum_{j=1}^{N_{i}^{b}} \underline{r}_{i j} \otimes \underline{F}_{i}^{j}\right)+\phi_{0} \mathbf{1}$

The Biot solid modulus, $N$, can be simulated in the NPTensemble applied to the solid phase(s), while pressure $p$ is applied to the entire solid boundary $\left(\partial V_{s}\right)$ utilizing Clapeyron's formula for linear elastic porous solids [40]:

$\frac{1}{\mathrm{~N}}=\frac{2 \Psi_{s}^{(\mathrm{NPT})}}{p^{2} V}-(\mathbf{1}-\mathbf{b}): \mathbb{S}:(\mathbf{1}-\mathbf{b})$

where $\Psi_{s}^{(\mathrm{NPT})}$ is the Helmholtz free energy of the solid phase and $\mathbb{S}=\mathbb{C}^{-1}$ is the effective fourth-order compliance tensor of the composite. Since clay is modeled in the reduced stiffness space, application of Eq. 27 is challenged given its dependence on effective compliance tensor, $\mathbb{S}$. To circumvent this, for the case of small displacement and infinitesimal strains, the discrete mass points can be meshed into volumetric finite elements of known relative nodal displacements, $\underline{\delta}_{i}=\underline{x}_{i}-\underline{X}_{i}$, where $\underline{x}_{i}$ and $\underline{X}_{i}$ denote position vectors of mass point $i$ in the reference and deformed configurations, respectively. For such an element, the strains can be calculated using the classical linear finite element approach (see, for instance [5]):

$\varepsilon_{i}=\left[B_{i j}\right]\left\{\xi_{j}\right\}$ 


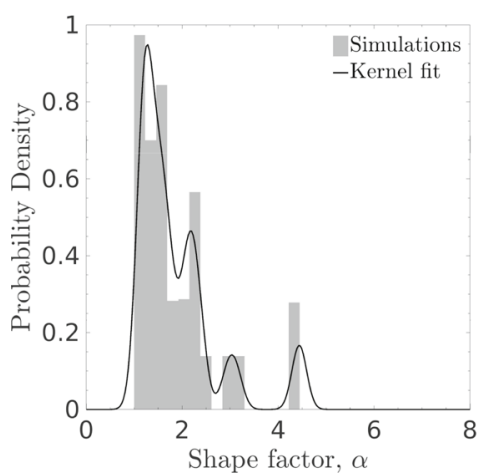

(a)

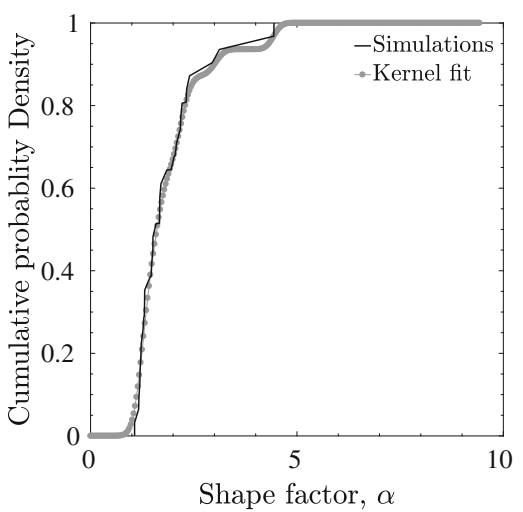

(c)

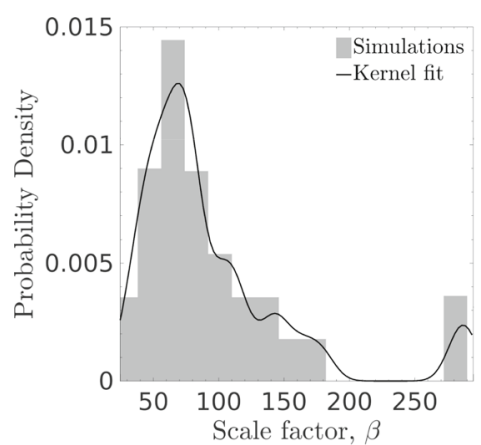

(e)

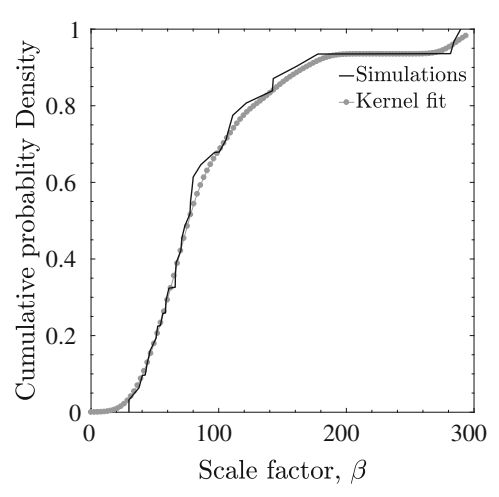

(g)

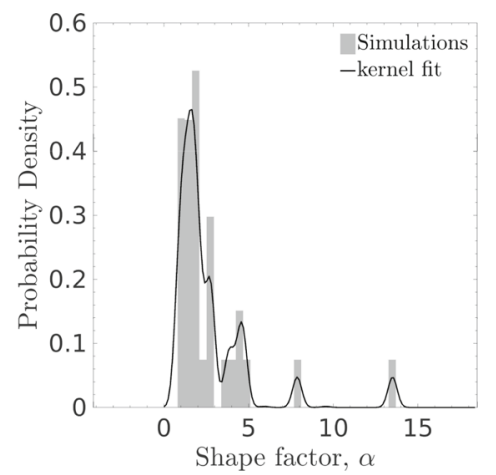

(b)

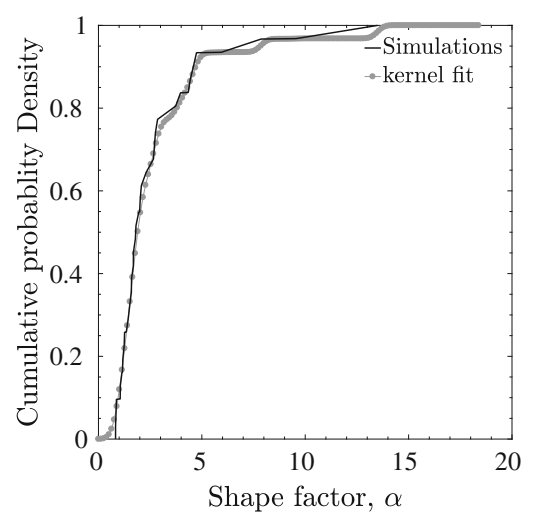

(d)

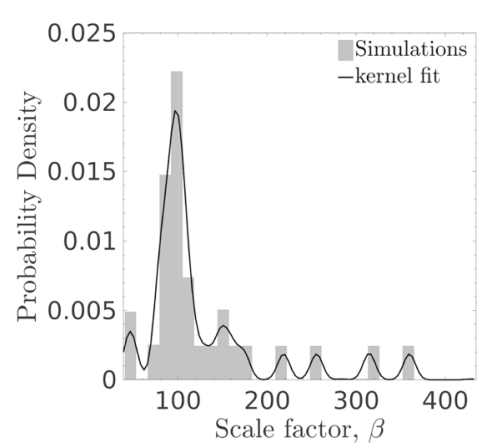

(f)

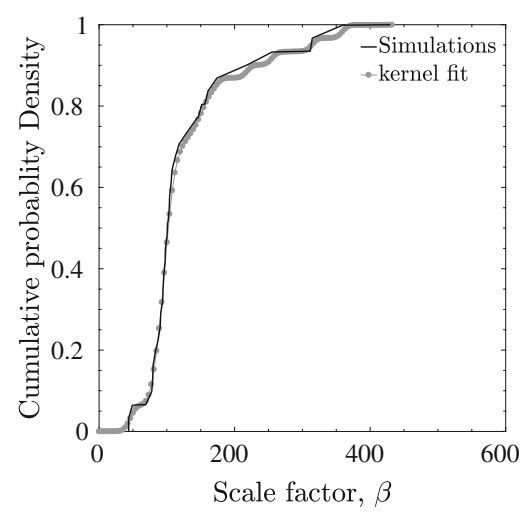

(h) 


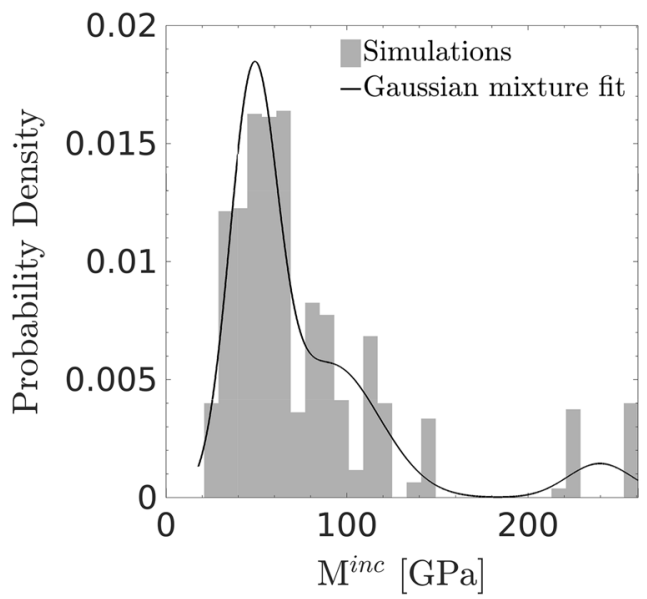

(a)

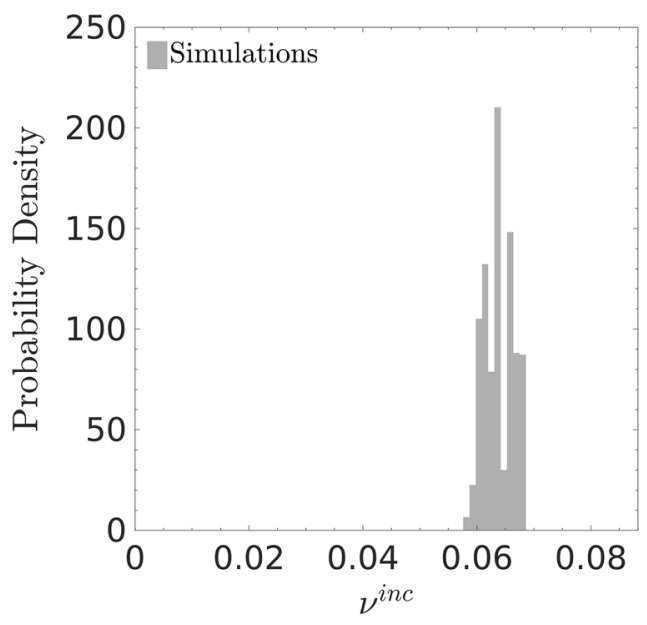

(c)

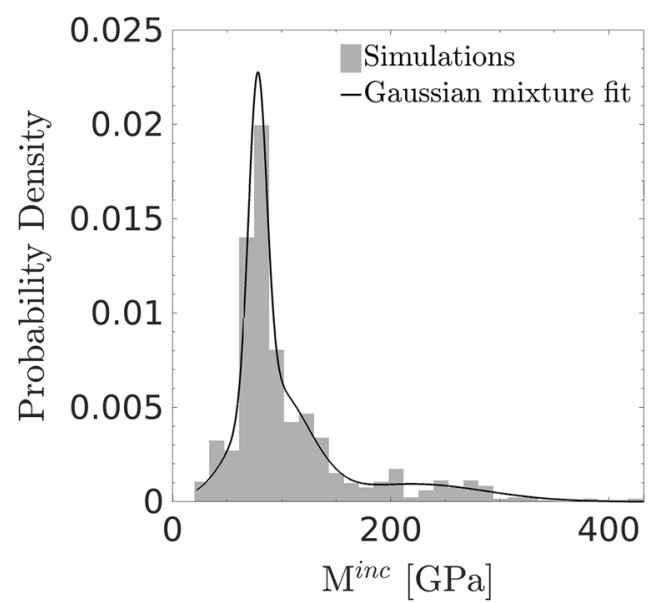

(b)

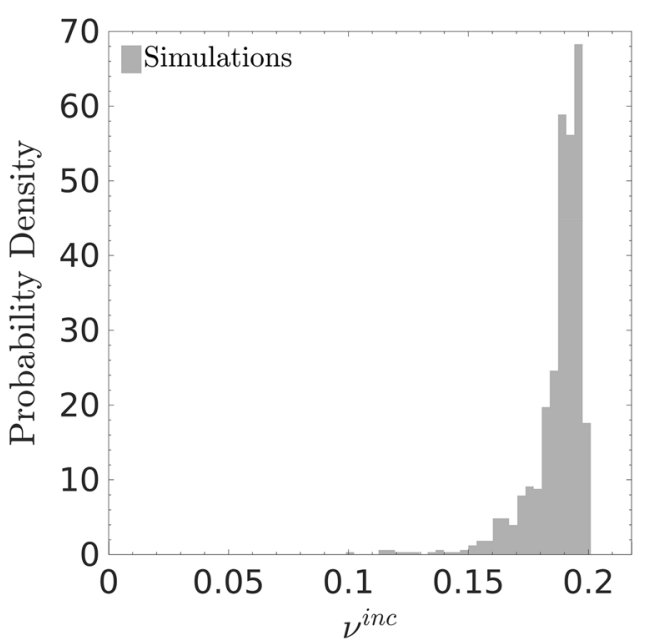

(d)

Fig. 11 Distributions of calibrated isotropic elasticity $\left(M^{\mathrm{inc}}, v^{\mathrm{inc}}\right)$ of the inclusions phase with three-component Gaussian mixture model fits for $M^{\text {inc }}$ for scan A $(\mathbf{a}, \mathbf{c})$ and scan B $(\mathbf{b}, \mathbf{d})$. See Table 2 for three-component Gaussian mixture model fitting parameters

Table 2 Three-component Gaussian mixture model fitting parameters for the isotropic indentation modulus, $M$, of the inclusion phase for scans A and B as shown in Fig. 11

\begin{tabular}{lllllll}
\hline & $w_{1}$ & $\mu_{1}$ & $w_{2}$ & $\mu_{2}$ & $w_{3}$ & $\mu_{3}$ \\
\hline Scan A & 0.56 & 48.17 & 0.06 & 239.77 & 0.38 & 91.15 \\
Scan B & 0.48 & 91.40 & 0.16 & 217.21 & 0.36 & 77.91
\end{tabular}

$\mu_{i}(\mathrm{GPa})$ denotes the mean of the $i$ th-component and $w_{i}$ the associated weight

where $\left[B_{i j}\right]$ is the element's strain-displacement matrix, $\left\{\xi_{j}\right\}$ is the vector of nodal displacements of the element, and $\varepsilon_{i}=\left\{\varepsilon_{11}, \varepsilon_{22}, \varepsilon_{33}, 2 \varepsilon_{12}, 2 \varepsilon_{23}, 2 \varepsilon_{31}\right\}^{T}$ is a vector representation of the linearized strain tensor representative of the strain state in the solid element. The advantage of this approach is its focus on solid bulk deformation, which is required for the determination of the poroelastic properties
Fig. 12 Distributions of calibrated quasi-transversely isotropic $\left(M_{1}\right.$, $\left.M_{3}, K_{\mathrm{VRH}}, G_{\mathrm{VRH}}\right)$ for the clay phase with normal fits for scan A (a, c, $\mathbf{e}, \mathbf{g})$ and scan B (b, d, f, h)

using the strain compatibility condition, i.e., $\operatorname{tr}(\mathbf{E})=\left(1-\phi_{0}\right)\langle\operatorname{tr}(\boldsymbol{\varepsilon})\rangle_{V_{\mathrm{s}}}+\left(\phi-\phi_{0}\right)$. Thus, in the $\mu \mathrm{VT}$ ensemble, an alternative access to the Biot modulus is obtained from:

$\left(\phi-\phi_{0}\right)^{(\mu \mathrm{VT})}=-\left(1-\phi_{0}\right)\langle\operatorname{tr}(\varepsilon)\rangle_{V_{s}}$

$\frac{1}{\mathrm{~N}}=\frac{1}{p}\left(\phi-\phi_{0}\right)^{(\mu \mathrm{VT})}$

The simulated Biot pore-pressure coefficients for scan A are plotted in Fig. 15 and for scan B in Fig. 16. Biot solid moduli for both scans are plotted in Fig 17. Lastly, the first two cumulants for the distribution of Biot poroelastic coefficients are summarized in Table 4. 


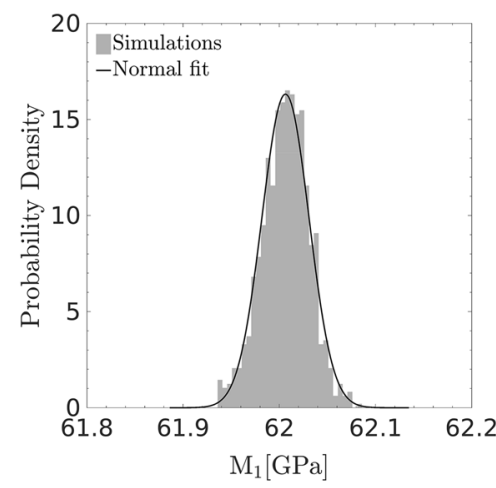

(a)

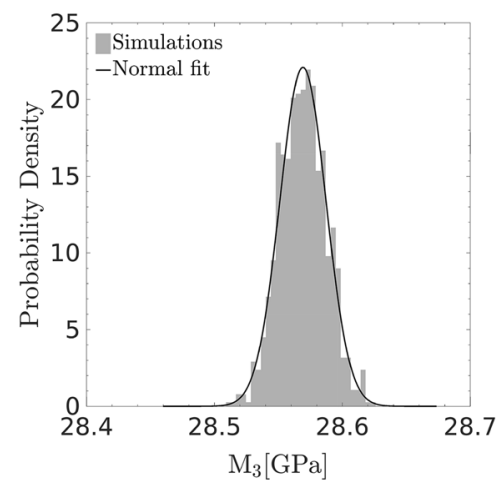

(c)

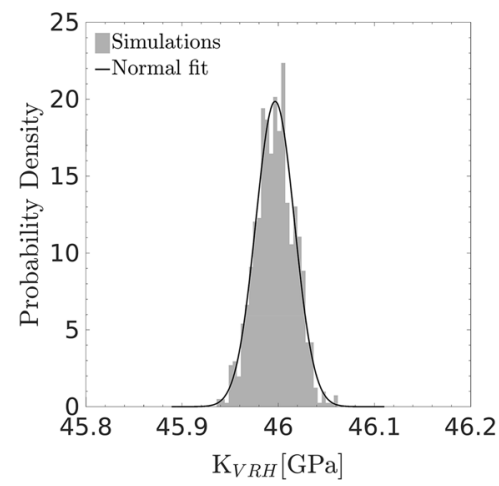

(e)

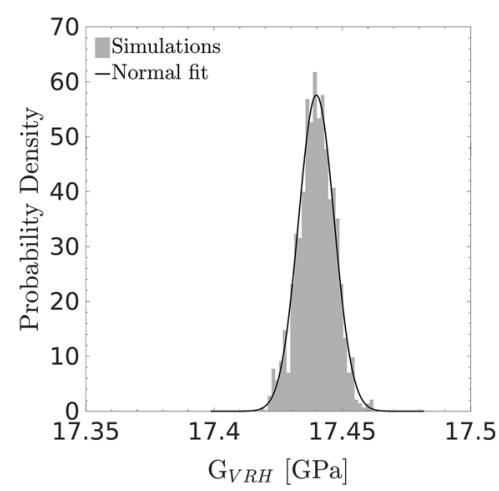

(g)

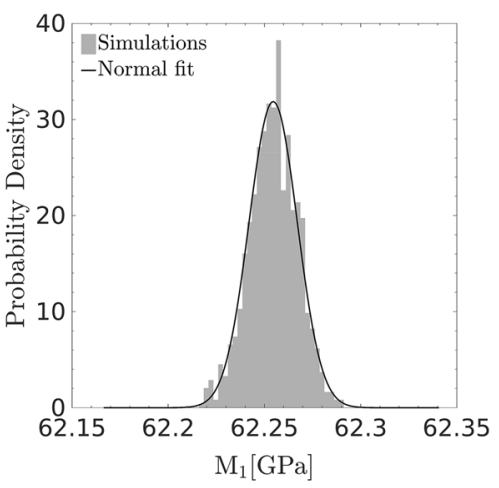

(b)

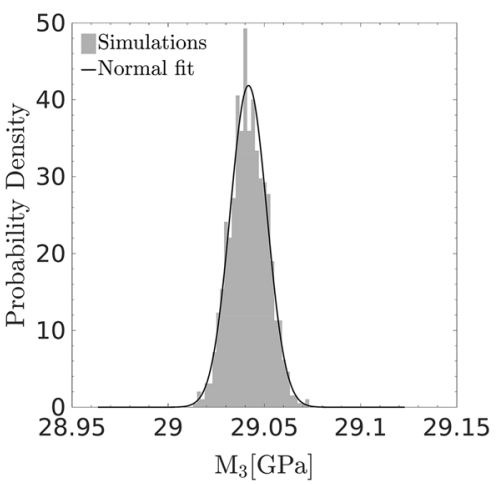

(d)

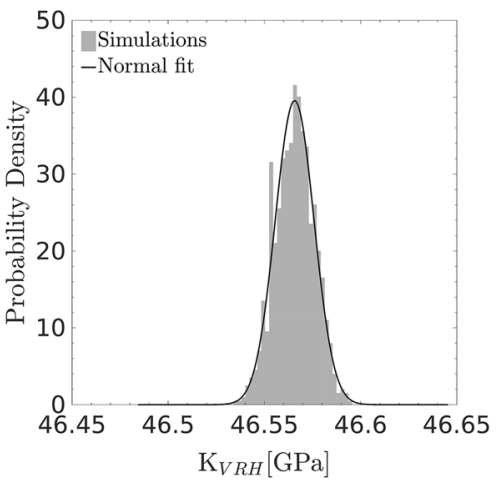

(f)

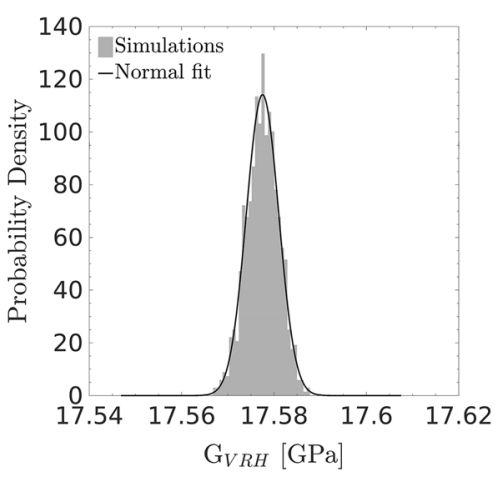

(h) 


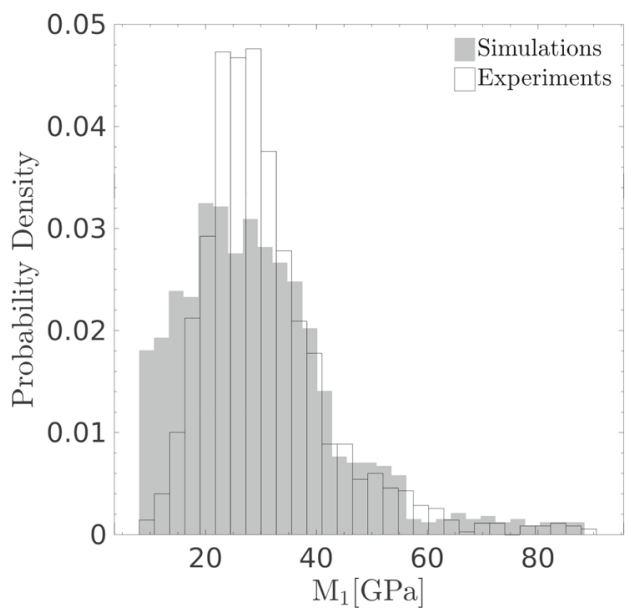

(a)

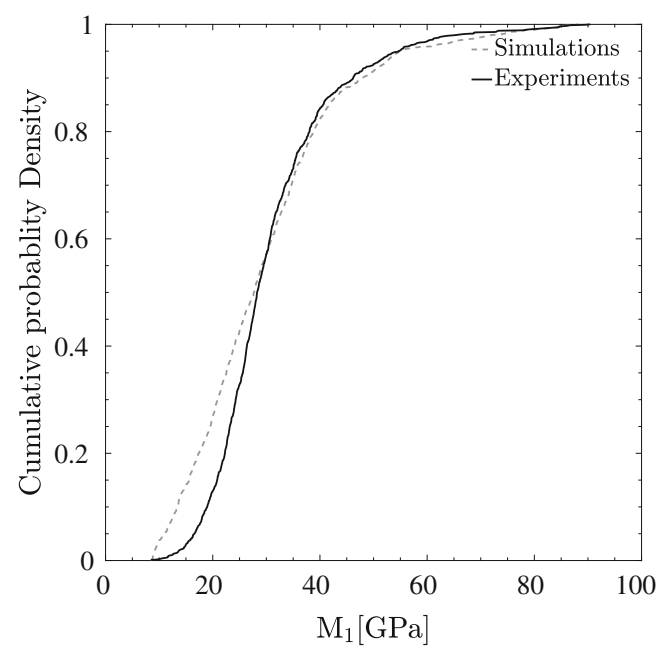

(c)

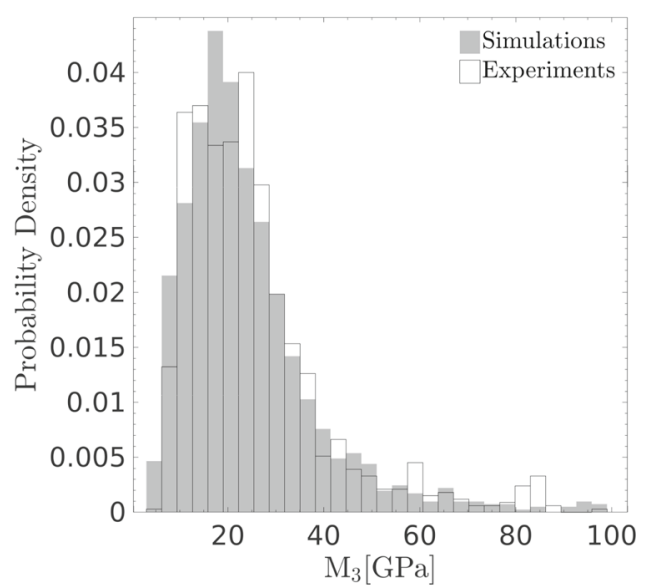

(b)

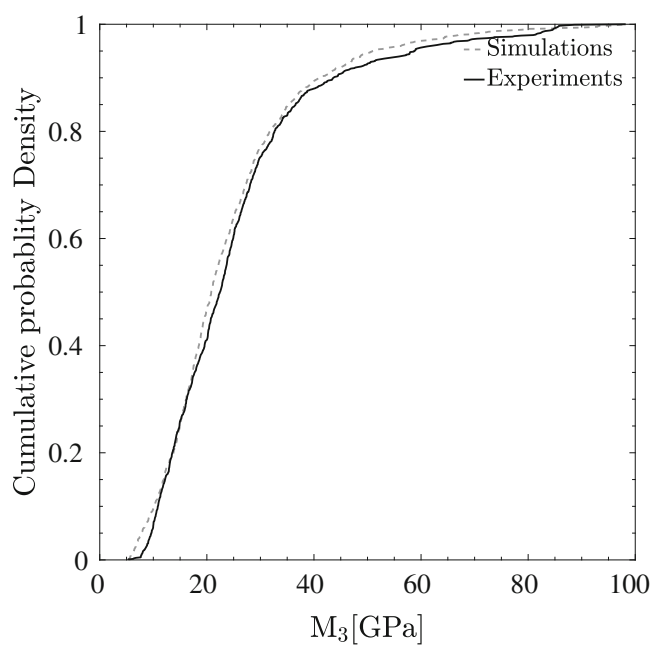

(d)

Fig. 13 Probability density (a, b) and cumulative probability density (c, d) of scan A for simulated indentation moduli and experimentally measured nanoindentation moduli using validation structure set in a forward application for validation

\subsection{Interface behavior sensitivity analysis}

The sensitivity of effective behavior, specifically simulated indentation moduli, on interface behavior is assessed by varying the interface behavior, i.e., energy parameters for the bond element connecting nodes $i$ and $j$ that belong to two different phases. To this end, interface properties are varied from $\min \left(\epsilon_{i j}^{(n, t)}, \epsilon_{j i}^{(n, t)}\right)$ to $\max \left(\epsilon_{i j}^{(n, t)}, \epsilon_{j i}^{(n, t)}\right)$ with results for CSS of scan B shown in Fig. 18, along with simulation results for interface behavior as defined by Eq. (15). The results highlight the low sensitivity of the computed outcome in this paper to the defined interface behavior in Eq. (15).

\subsection{Stress transmission}

Force flow and stress transmission through heterogeneous materials can provide a wealth of information regarding their microtexture (see for instance [34, 36, 46]). To explore this, first stress percolation in both scans due to an imposed uniaxial displacement (tension test) is studied. Then, for each scan, the local stresses are coarse-grained and their distributions plotted. Examining the response of coarse-grained stresses can be instrumental in determining the scale at which a representative elementary volume (rev) can be defined and thus continuum mechanics treatment can be applied. To achieve this, extracted sub-volumes are subjected to a uniaxial displacement while the simulation inputs are those obtained during calibration of effective interaction potentials of clay and inclusions phases. In addition, extracted sub-volumes of $150 \times 150 \times 150$ 


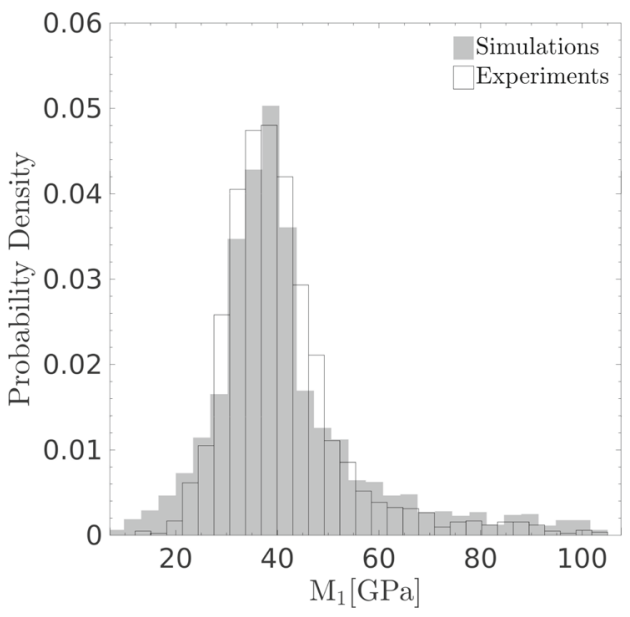

(a)

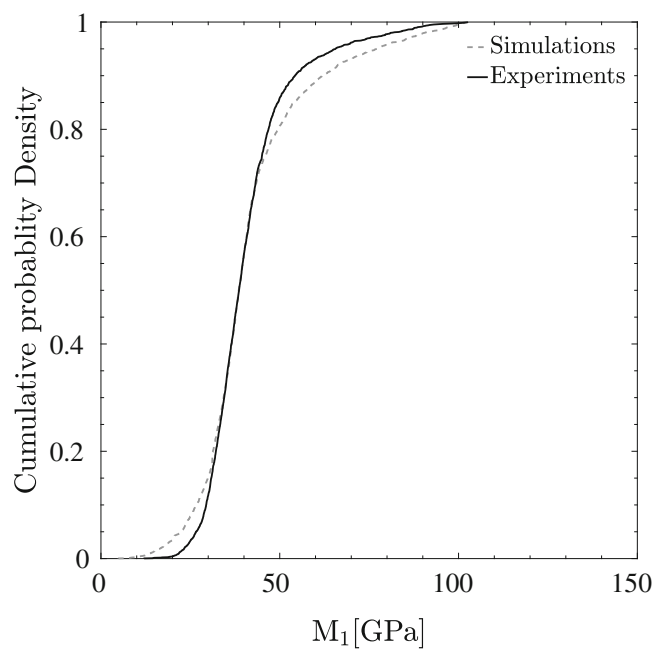

(c)

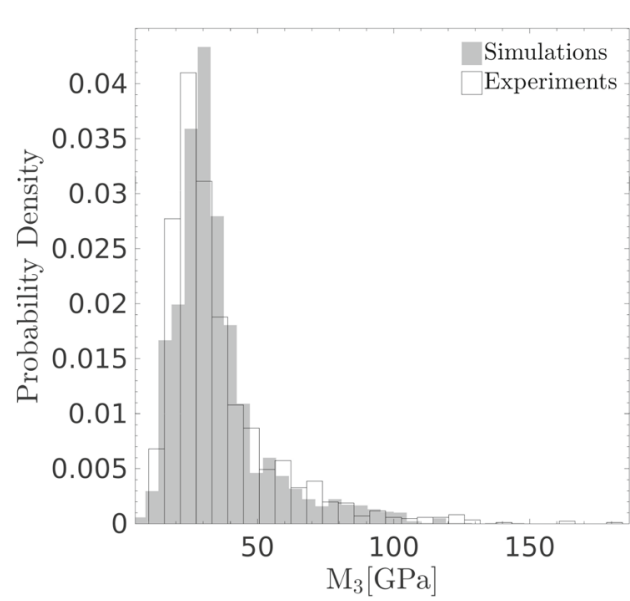

(b)

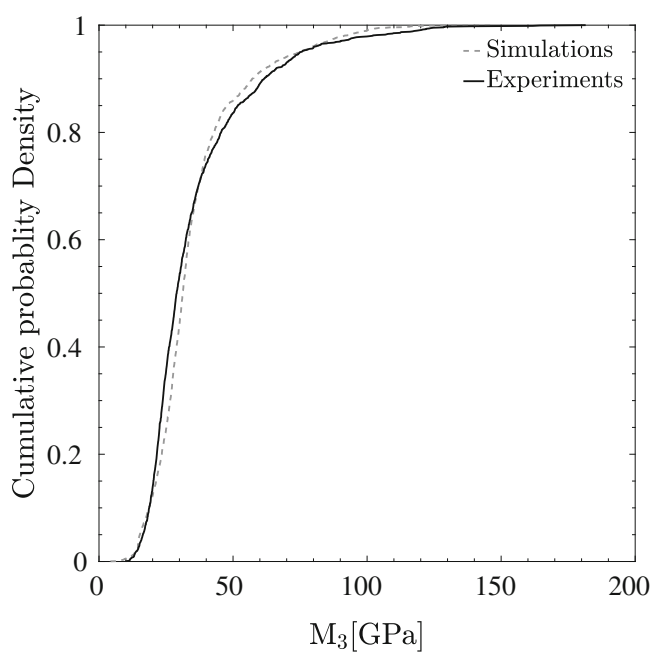

(d)

Fig. 14 Probability density (a, b) and cumulative probability density (c, d) of scan B for simulated indentation moduli and experimentally measured nanoindentation moduli using validation structure set in a forward application for validation

Table 3 First and second cumulants of the distributions of indentation moduli of validation structure set and experimentally measured

\begin{tabular}{lllllllll}
\hline & $\left\langle{ }^{\text {exp. }} M_{1}\right\rangle_{c}$ & $\left\langle{ }^{\text {exp. }} M_{1}^{2}\right\rangle_{c}$ & $\left\langle{ }^{\text {exp. }} M_{3}\right\rangle_{c}$ & $\left\langle{ }^{\text {exp. }} M_{3}^{2}\right\rangle_{c}$ & $\left\langle{ }^{\text {sim. }} M_{1}\right\rangle_{c}$ & $\left\langle{ }^{\text {sim. }} M_{1}^{2}\right\rangle_{c}$ & $\left\langle{ }^{\text {sim. }} M_{3}\right\rangle_{c}$ & $\left\langle{ }^{\text {sim. }} \cdot M_{3}^{2}\right\rangle_{c}$ \\
\hline Scan A & 30.933 & 144.816 & 25.483 & 235.540 & 29.591 & 207.437 & 24.130 \\
Scan B & 40.892 & 151.057 & 35.528 & 426.536 & 41.761 & 246.700 & 35.317 & 311.076 \\
\hline
\end{tabular}

voxels from each scan are characterized using the radial distribution function. The radial distribution function (only dependent on $\left.r=\left\|\underline{r}_{1}-\underline{r}_{2}\right\|\right)$ is used as an additional descriptor that carries information about position correlations in the system. It can be defined as:

$g(r)=\frac{\rho(r)}{\rho^{2}}$

where $\rho(r)$ is the local density, while $\rho$ is the average number density. The deviation of $g(r)$ from unity provides information with regard to spatial correlation between the particles, with unity corresponding to no spatial correlation [55]. The radial distribution functions for each phase are displayed in Fig. 19. The radial distribution functions show a strong correlation between the clay and pore phases in scan A, while for scan B, a strong correlation is observed between the inclusion and clay phases. Moreover, spatial distribution of clay phase in both scans are most correlated while spatial distribution of the inclusions and kerogen phases in both scans are least correlated. 


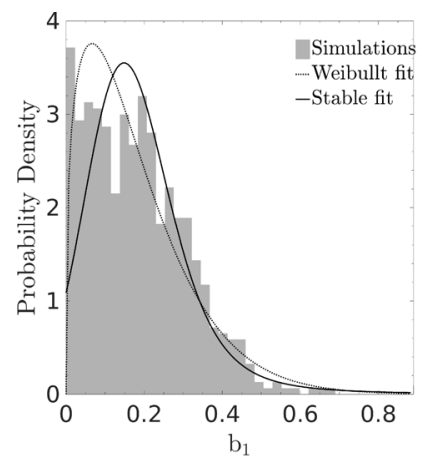

(a)

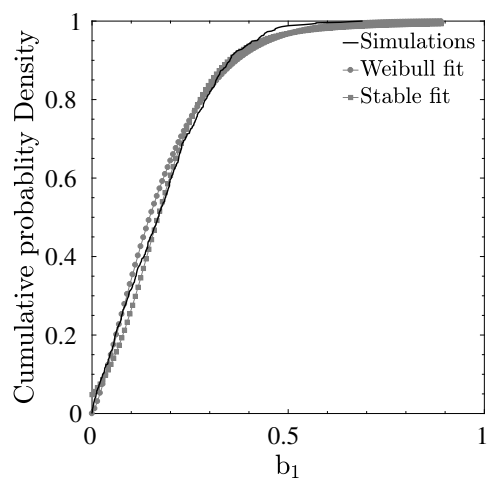

(d)

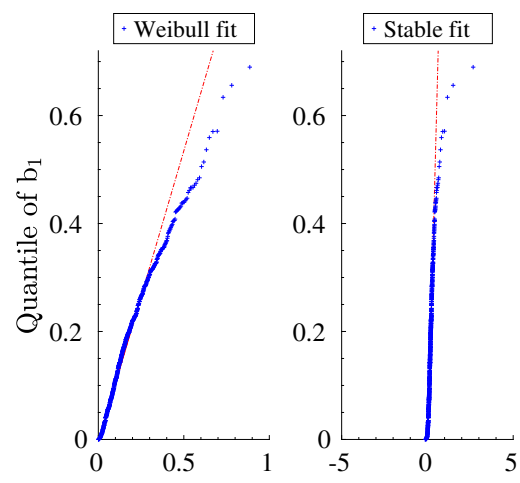

(g)

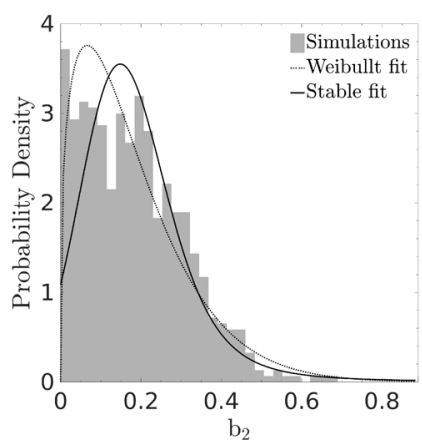

(b)

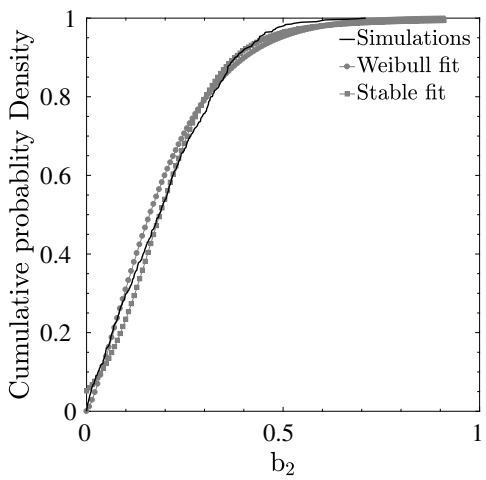

(e)
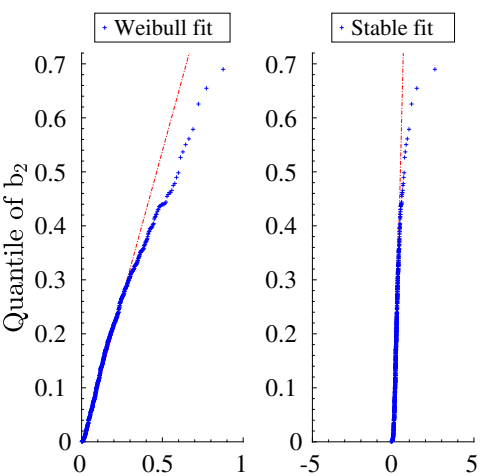

(h)

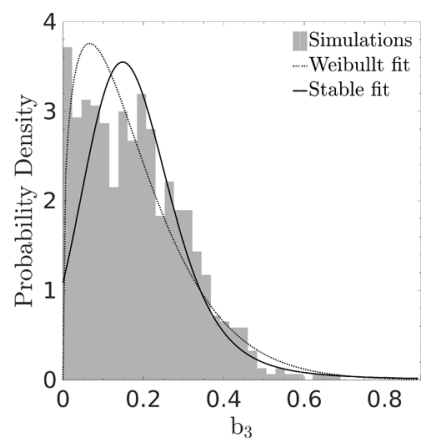

(c)

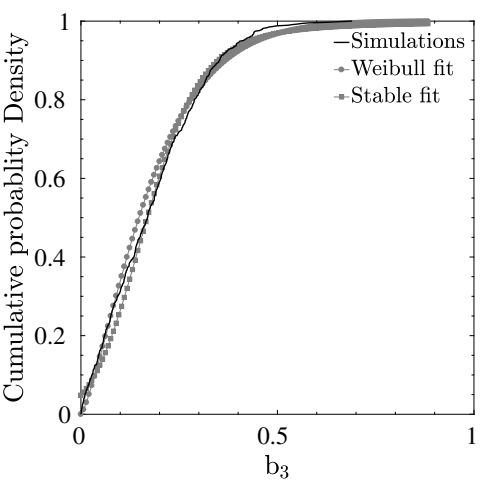

(f)
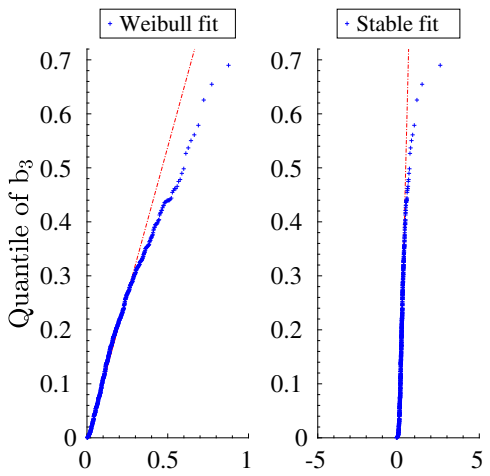

(i)

Fig. 15 Probability density (a-c), cumulative probability density $(\mathbf{d}-\mathbf{f})$ and quantile-quantile plots (g-i) for simulated Biot pore-pressure coefficients $b_{1}, b_{2}, b_{3}$ for scan A with fitted Weibull and Stable distributions

\subsubsection{Stress percolation and localization}

The percolating stress is defined as the stress threshold at which stresses form a continuous path through the sample in the direction of the loading (displacement or traction). This can be found by thresholding the stress, starting from highest stress in the sample and continuously monitoring the localization of the stresses until a continuous path of stresses greater than or equal to the threshold is formed in the direction of the imposed loading. A continuous path is defined as such that each voxel satisfying the stress thresholding criteria has a neighboring voxel, based on 26connectivity, that also meets the stress threshold, $\sigma$ th.. Subvolumes of $150 \times 150 \times 150$ voxels are extracted from each scan. A uniaxial displacement in the form of $\underline{\xi}^{d}=$ $\xi^{d} \underline{e}_{i}$ for $i \in\{1,2,3\}$ is imposed on the extracted sub-volumes and for five realizations of the obtained Weibull distributions for the inclusions effective interaction potentials. As an example, percolation path for one of the realizations is shown in Fig. 20. A quantity of interest, 


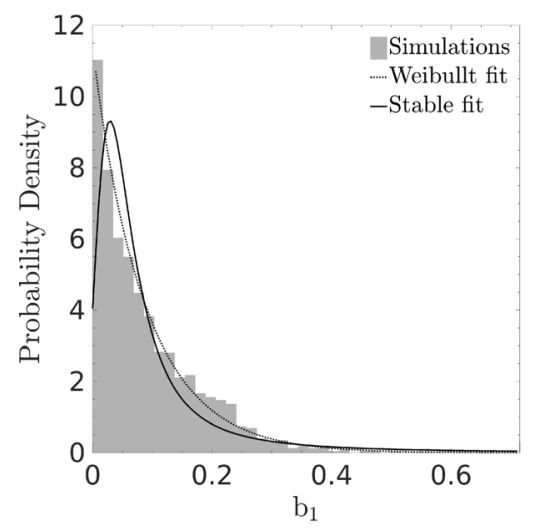

(a)

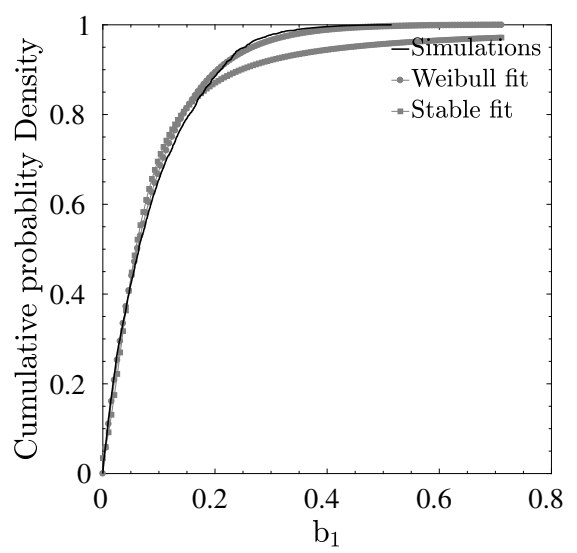

(d)

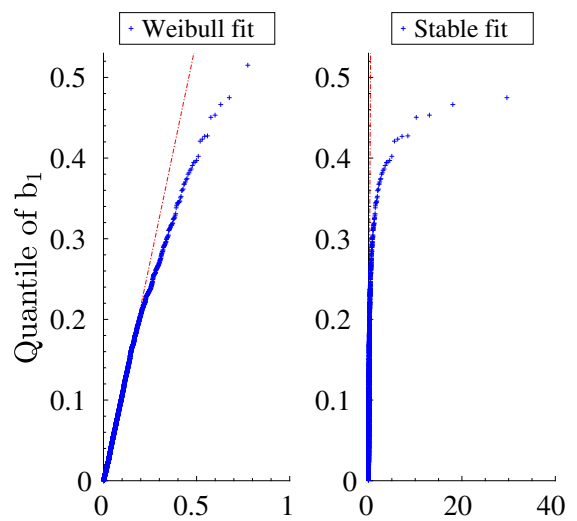

(g)

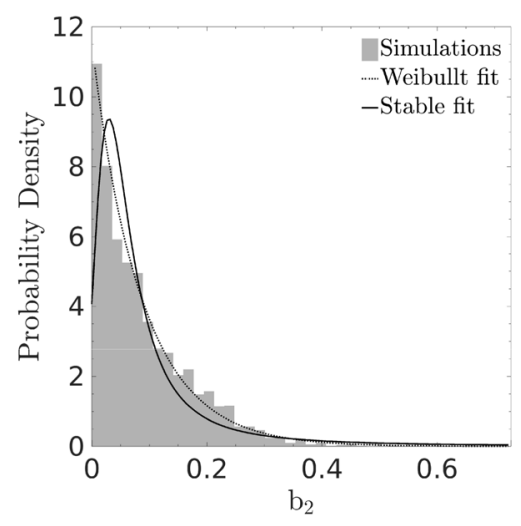

(b)

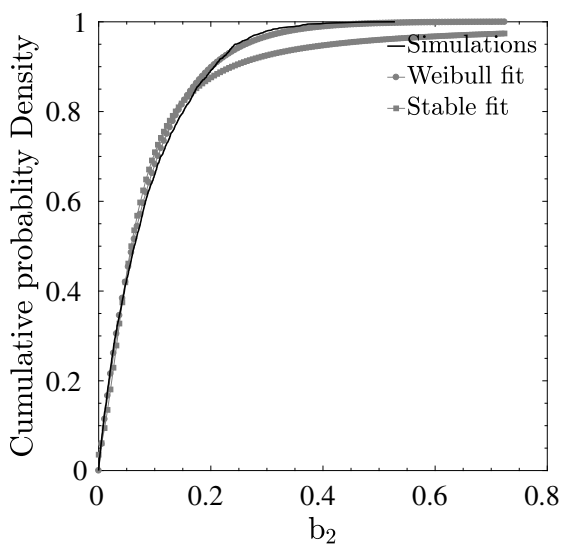

(e)
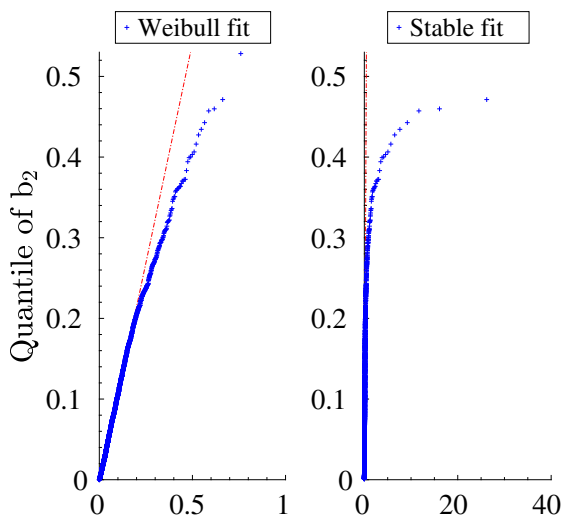

(h)

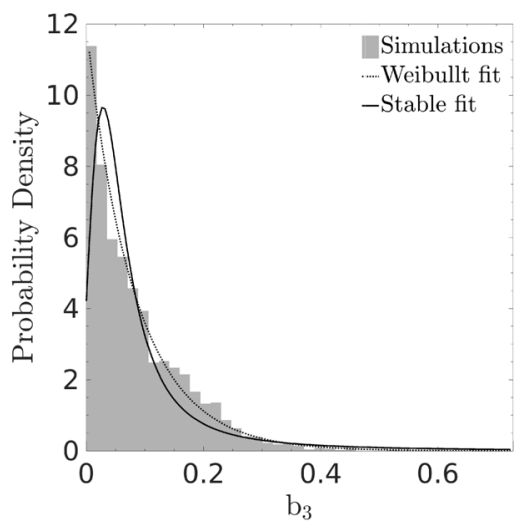

(c)

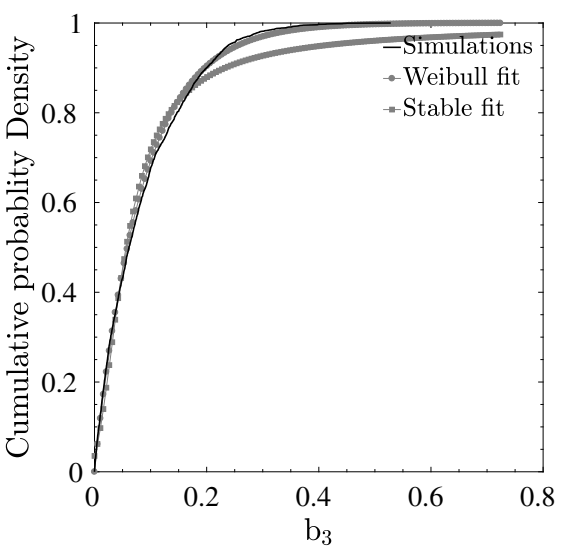

(f)
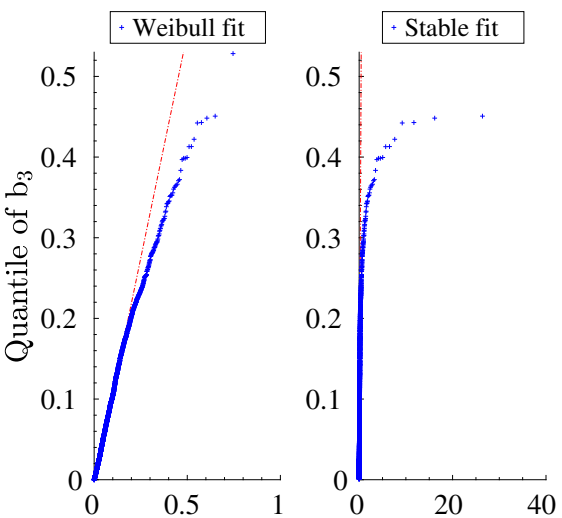

(i)

Fig. 16 Probability density $(\mathbf{a}-\mathbf{c})$, cumulative probability density $(\mathbf{d}-\mathbf{f})$ and quantile-quantile plots $(\mathbf{g}-\mathbf{i})$ for simulated Biot pore-pressure coefficients $b_{1}, b_{2}, b_{3}$ for scan B with fitted Weibull and Stable distributions

highlighting the underlying microtextural features of a highly heterogeneous media, is the ratio of average stresses in the direction of imposed load to the stress threshold. For the cases examined, this ratio is summarized in Table 5. In addition, the radial distribution function for stresses greater than the percolating stress, for all five realizations for clay and inclusions phases' elasticity, is shown in Fig. 21. The results highlight the important role of local stiffness variations on percolating stress paths with the $\underline{e}_{1}$ direction (parallel to the bedding planes) in scan A least affected and the $\underline{e}_{3}$ direction (perpendicular to the bedding planes) in scan $B$ most affected by such variations. 


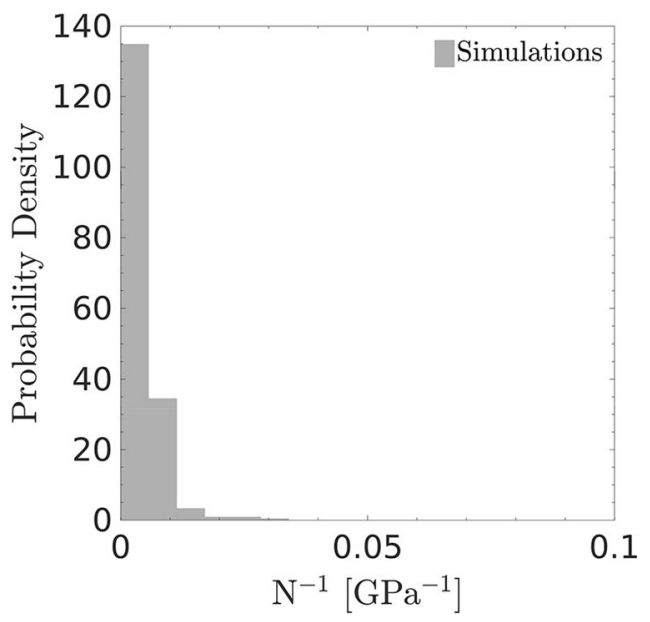

(a)

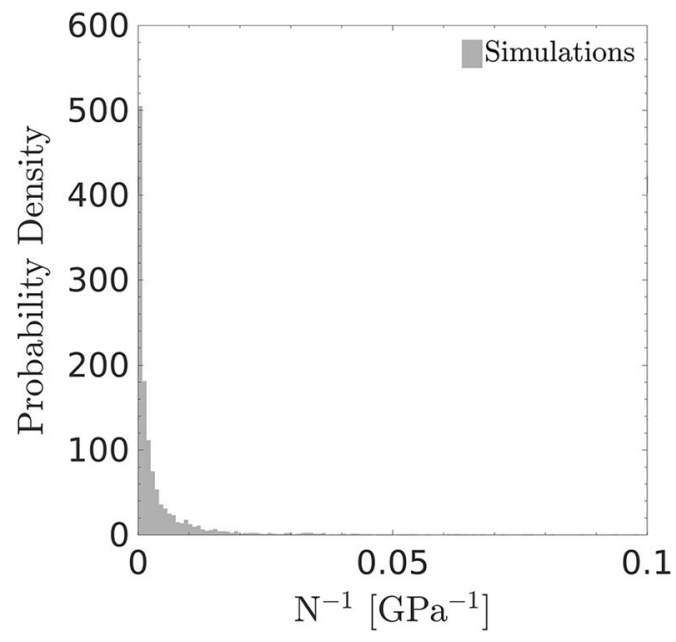

(b)

Fig. 17 Distributions for Biot solid modulus for scan A (a) and scan B (b)

Table 4 First and second cumulants of the distributions for Biot poroelastic coefficients

\begin{tabular}{|c|c|c|c|c|c|c|c|c|}
\hline & $\left\langle b_{1}\right\rangle_{c}$ & $\left\langle b_{1}^{2}\right\rangle_{c}$ & $\left\langle b_{2}\right\rangle_{c}$ & $\left\langle b_{2}^{2}\right\rangle_{c}$ & $\left\langle b_{3}\right\rangle_{c}$ & $\left\langle b_{3}^{2}\right\rangle_{c}$ & $\left\langle N^{-1}\right\rangle_{c}$ & $\left\langle\left(N^{-1}\right)^{2}\right\rangle_{c}$ \\
\hline Scan A & 0.182 & 0.016 & 0.198 & 0.019 & 0.182 & 0.016 & 0.0062 & 0.0019 \\
\hline Scan B & 0.089 & 0.007 & 0.087 & 0.007 & 0.085 & 0.006 & 0.0043 & $8.9659 \times 10^{-5}$ \\
\hline
\end{tabular}

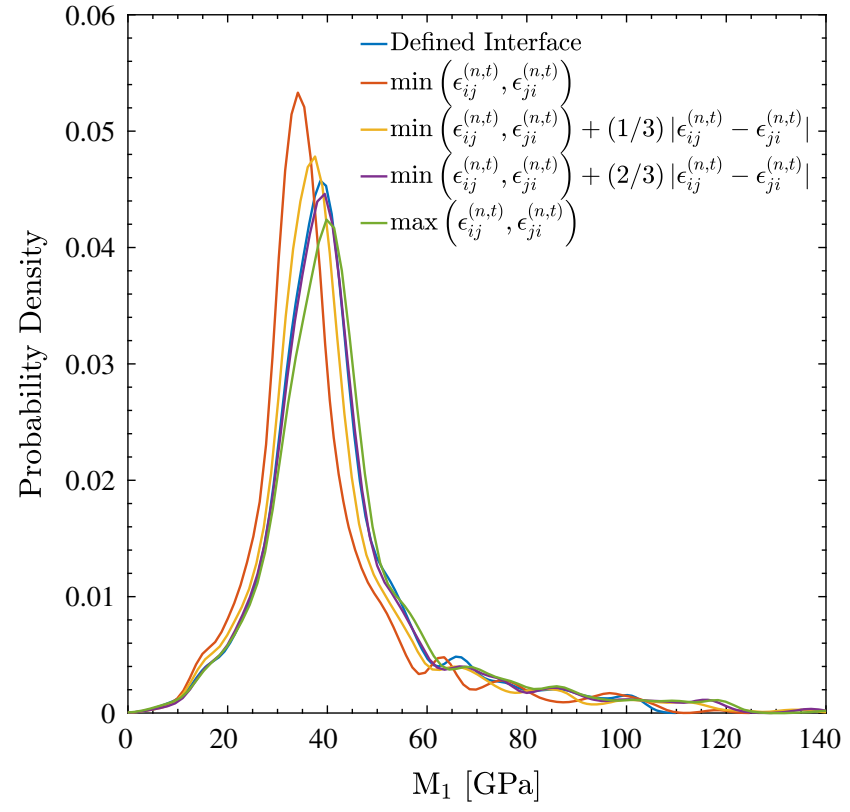

(a)

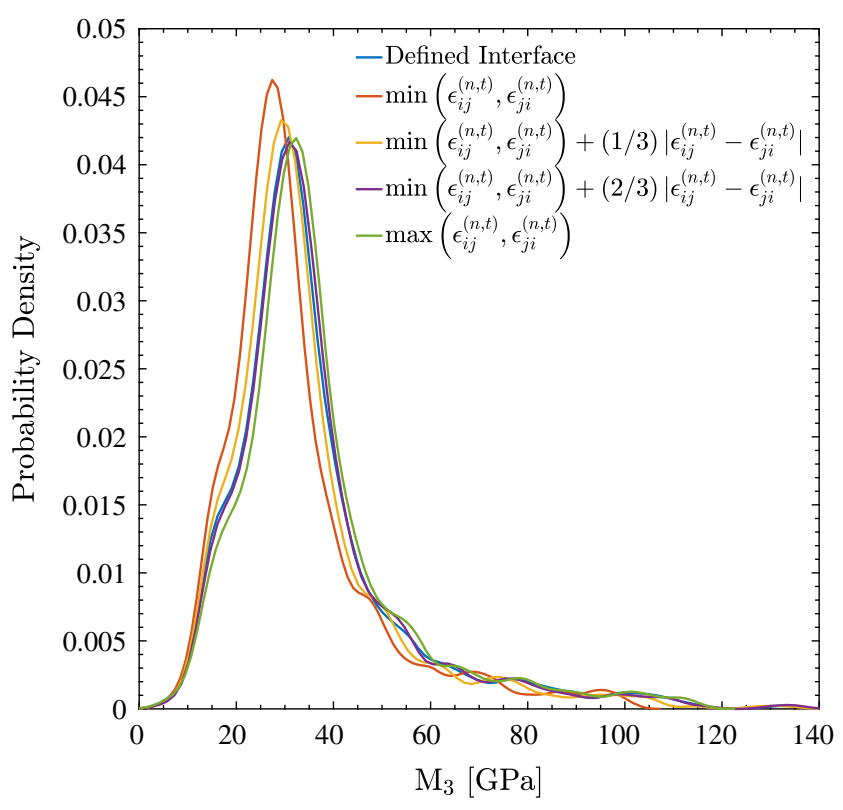

(b)

Fig. 18 Distributions for simulated indentation moduli, $M_{1}$ as shown in $\mathbf{a}$ and $M_{3}$ as shown in $\mathbf{b}$, for different interface models

\subsubsection{Stress coarse-graining}

Sub-volumes of size $300 \times 300 \times 300$ voxels are extracted for both scans and subjected to a uniaxial displacement of the form $\underline{\xi}^{d}=\xi^{d} \underline{e}_{i}$ for $i \in\{1,2,3\}$ and for five realizations of the obtained Weibull distributions for the inclusions effective interaction potentials. The local stresses are coarse-grained by averaging them over sub-volumes of increasing length, $\lambda / a_{0}(=1)$ while applying a periodic boundary condition. The resulting distributions plotted for 


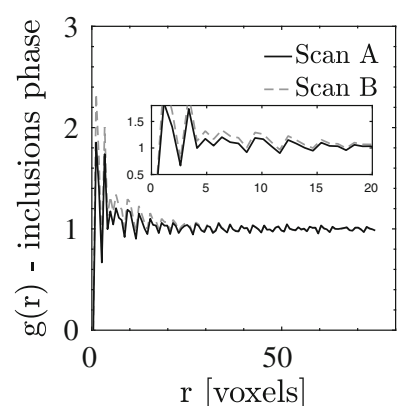

(a)

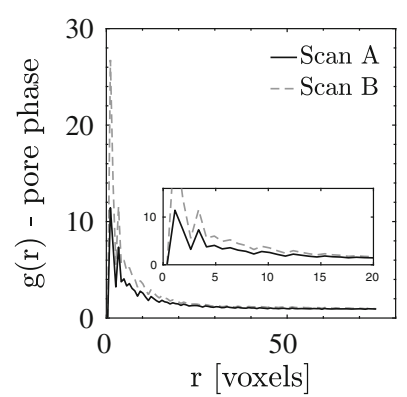

(c)

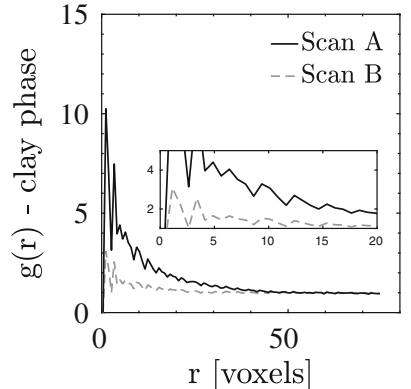

(b)

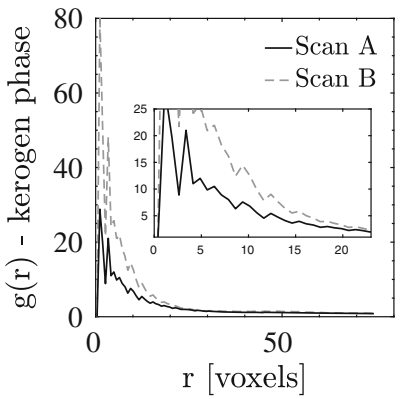

(d)

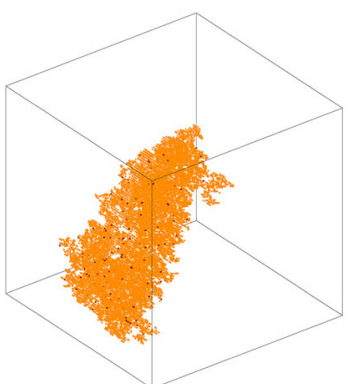

(a)

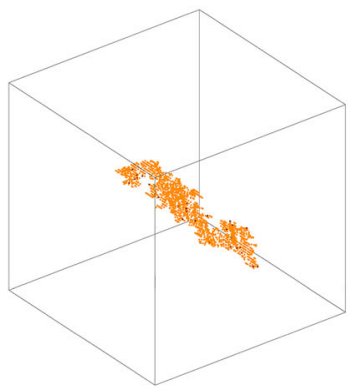

(c)

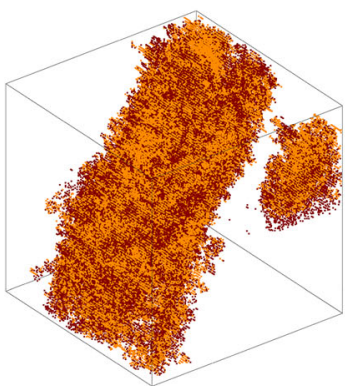

(e)

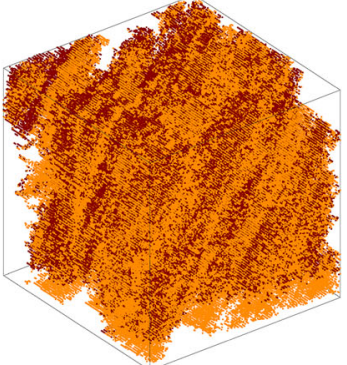

(b)

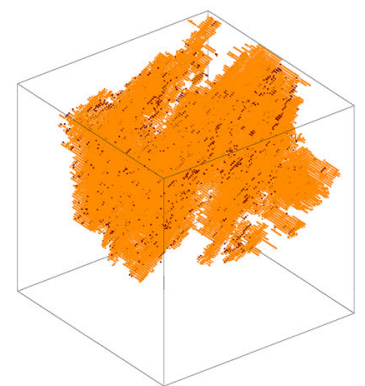

(d)

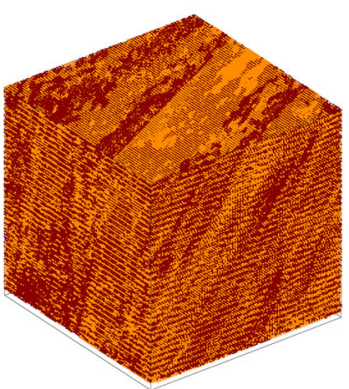

(f) grained samples falls below 200 and thus excluded for statistical analysis. For $\lambda=50$, representing 216 samples, both scans display multimodal behaviors though with significantly less noise compared to the cases for $\lambda<50$. Such multimodal behavior is a reflection of existence of competing load-bearing phases at this coarse-graining length scale. Additionally, the dependency of the results on different stiffness realizations should be noted. For example for $\lambda=50$, both multimodal and bimodal behaviors can be observed.

\section{Discussions}

It is shown that capturing the heavy-tailed distributions of experimentally measured indentation moduli requires introducing spatial fluctuations in the effective interaction potential of the inclusions and clay phases. For the inclusions phase, this is achieved by modeling these spatial variations with a continuous univariate Weibull

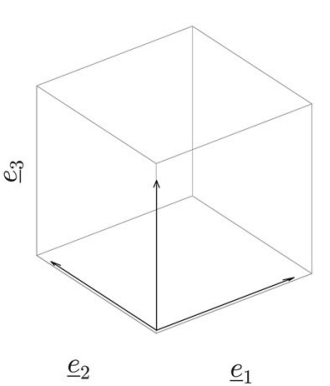

(g)

Fig. 20 Percolating stress path in $\underline{e}_{1}$ and for scan A (a), for scan B (b), in $\underline{e}_{2}$ and for scan A (c) and scan B (d), in $\underline{e}_{3}$ and for scan A (e) and scan B (f). Colors red and orange indicate inclusions and clay phases, respectively. g Defines the coordinate system (color figure online)

distribution while for the clay phase, small spatially dependent fluctuations are introduced following Gaussian distributions. The PDFs for calibrated elasticity of the inclusions phases for each scan, as shown in Fig. 11, reflect 
Table 5 Ratio of mean stresses to stress threshold for a given direction of applied load

\begin{tabular}{llll}
\hline & $\left\langle\sigma_{11}\right\rangle / \sigma_{11}^{\text {th. }}$ & $\left\langle\sigma_{22}\right\rangle / \sigma_{22}^{\text {th. }}$ & $\left\langle\sigma_{33}\right\rangle / \sigma_{33}^{\text {th. }}$ \\
\hline Scan A & $2.88-3.87$ & $3.54-4.51$ & $2.78-3.13$ \\
Scan B & $1.28-1.69$ & $1.34-1.53$ & $0.93-1.16$ \\
\hline
\end{tabular}

the range of elasticity represented by the diverse group of the inorganic minerals (see Fig. 7 for compositional piecharts) considered to be part of the inclusions phase. This is evident by the multimodal nature of these PDFs. The fitted three-component Gaussian mixture model parameters, as outlined in Table 2, reflect the composition of the inclusions phase as displayed in Fig. 7. With a focus on isotropic indentation modulus, the typical elasticity values reported in the literature (see, e.g., [39]) are $\mathrm{M}^{\text {Quartz }}=95.28 \mathrm{GPa}, \quad \mathrm{M}^{\text {Albite }} \approx \mathrm{M}^{\text {Feldspar }}=78.51 \mathrm{GPa}$, $\mathrm{M}^{\text {Pyrite }}=313.30 \mathrm{GPa}, \quad \mathrm{M}^{\text {Calcite }}=93.91 \mathrm{GPa} \quad$ and $\mathrm{M}^{\text {Dolomite }}=127.71 \mathrm{GPa}$ which are very comparable to values reported in Table 2. This convergence of XRD data and calibrated effective interaction potential of inclusions phase from laboratory-measured nanoindentation moduli highlights the utility of modeling spatial fluctuations of inclusion elasticity using a two-parameter Weibull model. The small range of obtained Poisson's ratio can be attributed to maintain a constant ratio between $\epsilon_{i}^{(n, t)}$ in different directions, as outlined in Eq. (19). Relaxing this condition would result into higher spatial fluctuations in Poisson's ratio while increasing the degrees of freedom associated with the calibration procedure. Furthermore, additional refinements can be considered in the future by introducing multivariate continuous random variables with spatial correlations (see, e.g., [58]). The local variations of mechanical properties, spatially and within a phase, are observed experimentally in a range of materials with some studies attributing enhanced mechanical behavior to such spatial variations $[18,52,61]$ while more recently highlighting the role of stiffness heterogeneity in cell mechanics [8].

The experimentally measured indentation moduli can be modeled by both lognormal and Stable distributions as shown in Figs. 5 and 6. The quantile-quantile plots suggest Stable distribution to be a slightly better model to fit the data given the deviations at the distribution tails from the models. In fact, it is well known that Stable distributions

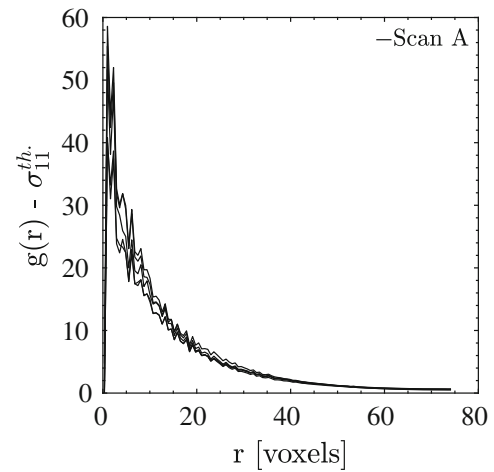

(a)

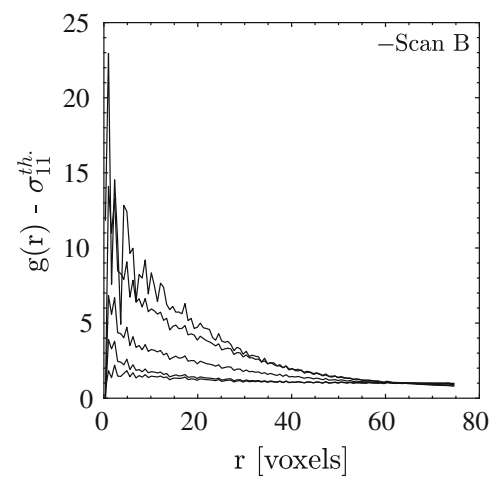

(d)

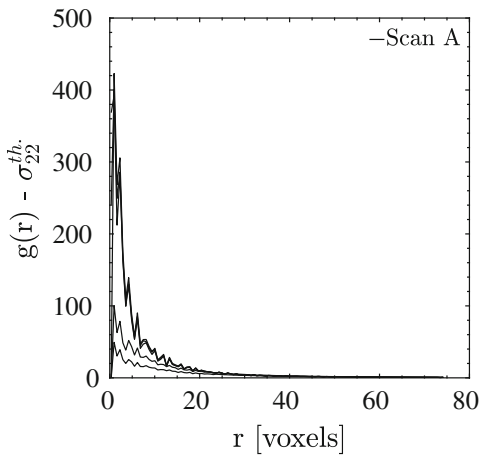

(b)

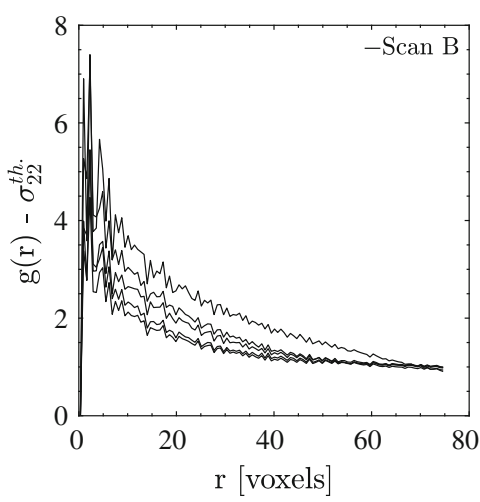

(e)

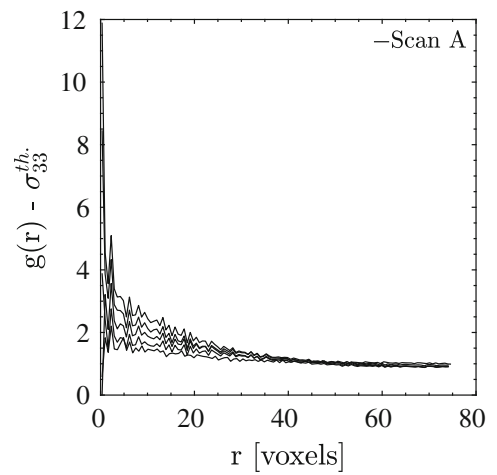

(c)

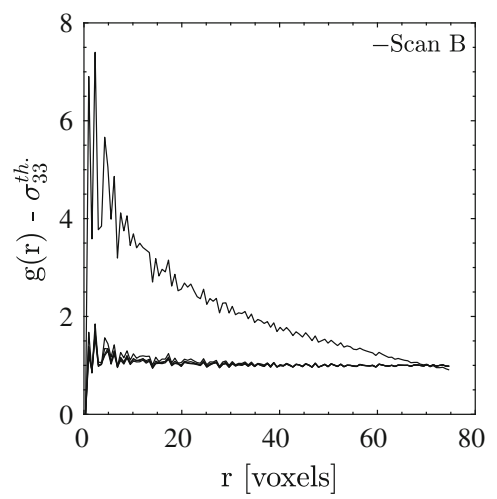

(f)

Fig. 21 Radial distribution function for the percolated stress path due to imposed uniaxial displacement in different directions for scan A (ac) and scan B (d-f) 


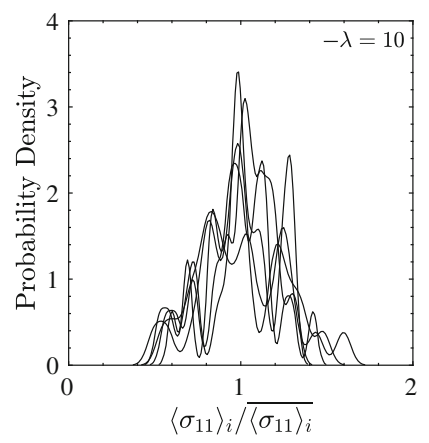

(a)

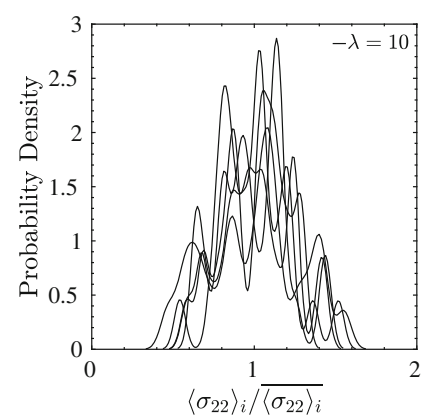

(e)

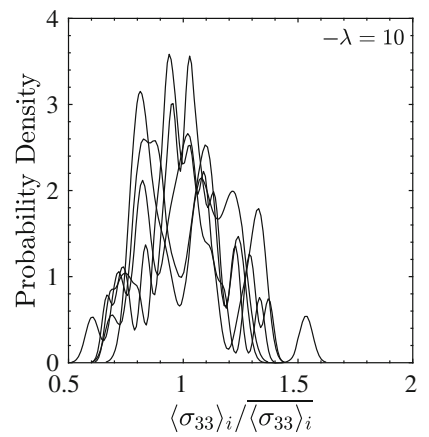

(i)

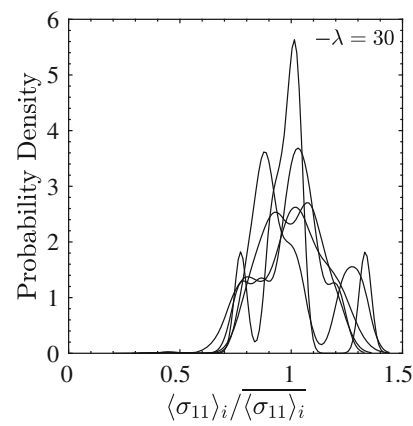

(b)

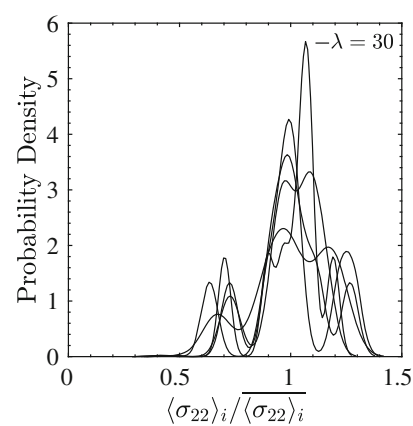

(f)

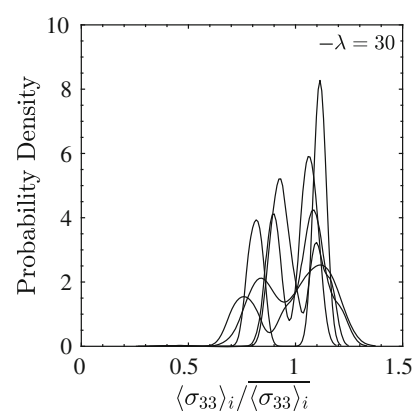

(j)

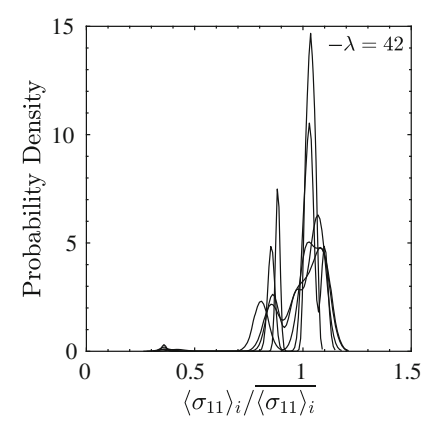

(c)

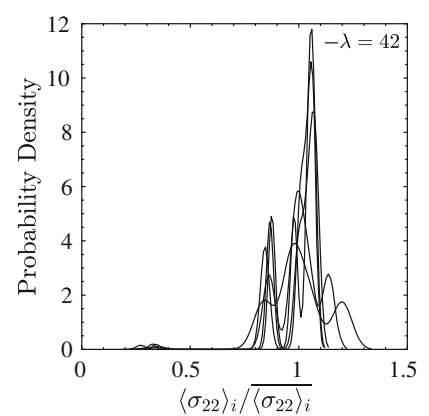

(g)

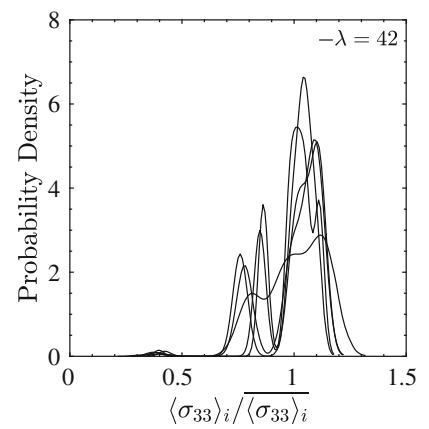

(k)

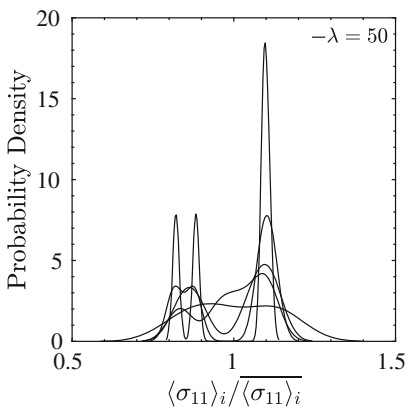

(d)

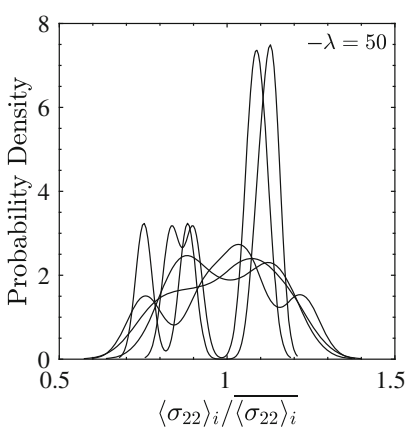

(h)

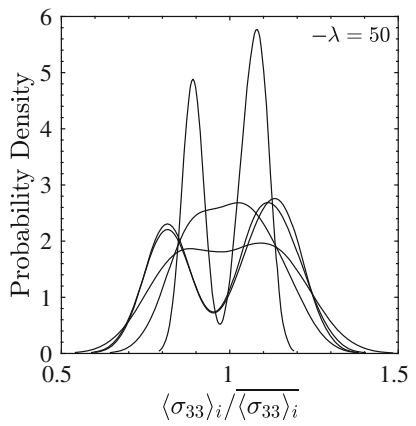

(I)

Fig. 22 Coarse-grained stresses for different $\lambda$ for scan A with five realizations for inclusions stiffness, labeled as cases $A-E$. a-d correspond to coarse-grained stresses, $\sigma_{11}$ due to $\underline{\xi}^{d}=\xi^{d} \underline{e}_{1} ; \mathbf{e}-\mathbf{h}$ correspond to coarse-grained stresses, $\sigma_{22}$ due to $\underline{\xi}^{d}=\xi^{d} \underline{e}_{2}$ and $\mathbf{i}-\mathbf{l}$ correspond to coarsegrained stresses, $\sigma_{33}$ due to $\underline{\xi}^{d}=\xi^{d} \underline{e}_{3}$

arise from interaction of very wide-PDFs [30], in this case a manifestation of the highly heterogeneous nature of organic-rich shales even at very small length scales (see, e.g., [2]) from the generalized central limit theorem [23]. This observation can also be extended to the simulated effective Biot pore-pressure coefficients, as shown in Figs. 15 and 16, with the simulated data captured well with Weibull and Stable distributions, except for the heavy-tails. The simulated Biot solid moduli do not follow any parametric distributions, and thus, only the simulated results are shown as PDFs in Fig. 17 for both scans. Notable from these PDFs, simulated Biot solid moduli for scan B cover a wider range compared to scan A. The first two cumulants of the simulated Biot poroelastic coefficients are summarized in Table 4. The first cumulants suggest no significant anisotropy given the length scale associated with simulated sub-volumes. It should be noted that, on average, the Biot poroelastic coefficients of scan A are two times greater than that of scan B. This is partly due to scan A having a higher porosity, on average, compared to scan B as shown in Figs. 2 and 3.

The distribution of stresses as a result of an imposed uniaxial displacement was also studied. It was shown that stresses percolated at a higher threshold in scan A compared to scan B as summarized in Table 5. The percolation path as shown in Fig. 20 points at the underlying 


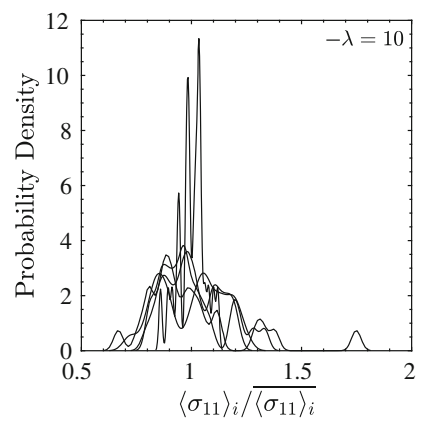

(a)

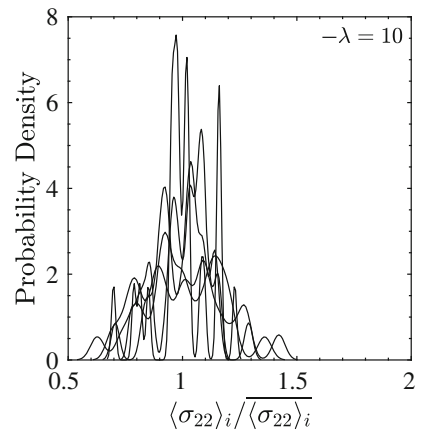

(e)

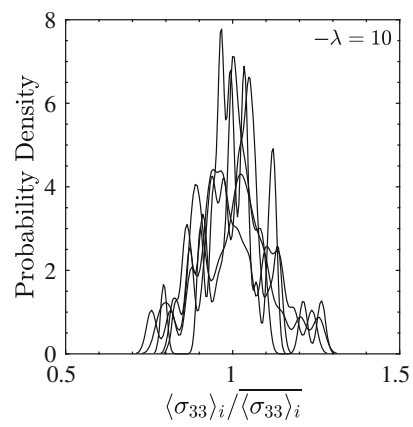

(i)

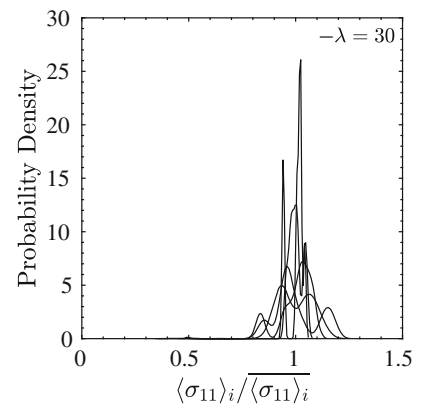

(b)

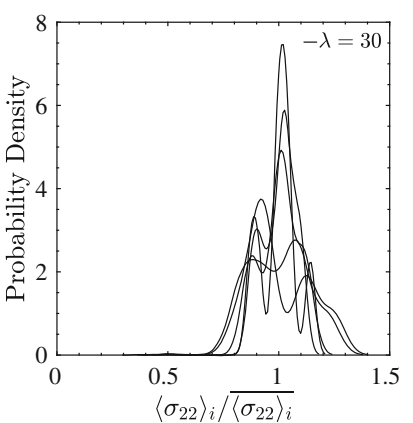

(f)

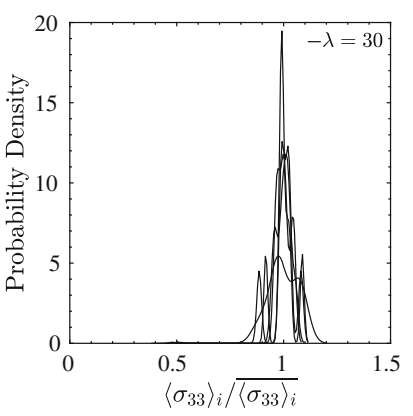

(j)

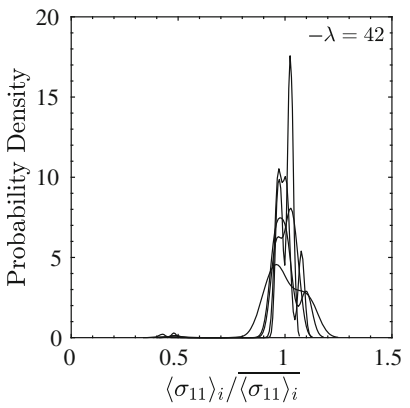

(c)

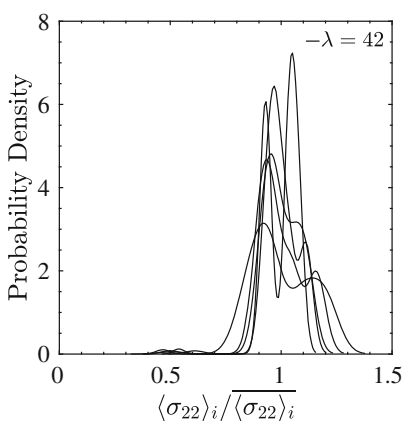

(g)

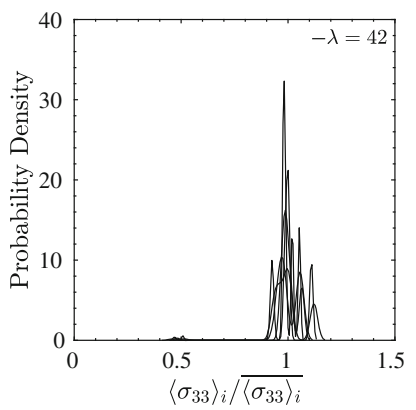

(k)

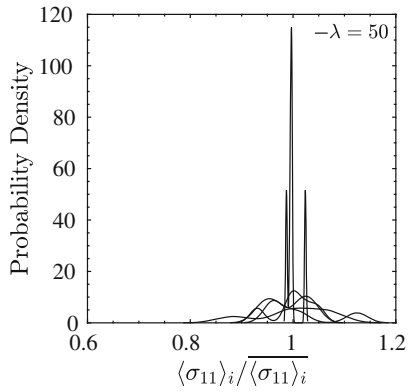

(d)

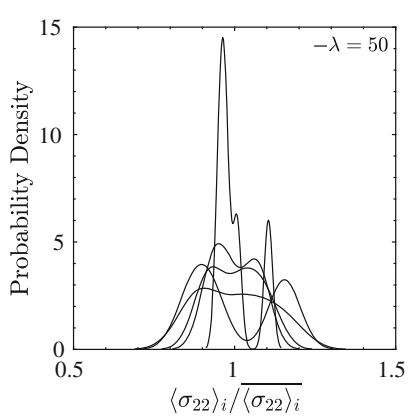

(h)

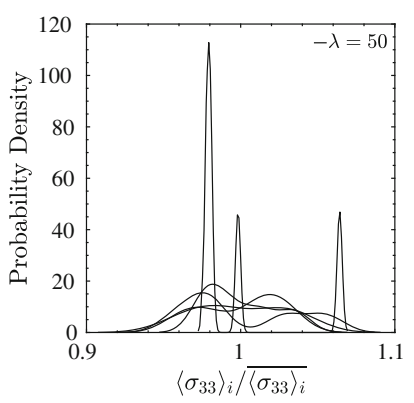

(l)

Fig. 23 Coarse-grained stresses for different $\lambda$ for scan B with five realizations for inclusions stiffness, labeled as cases $A-E$. a-d correspond to coarse-grained stresses, $\sigma_{11}$ due to $\underline{\xi}^{d}=\xi^{d} \underline{e}_{1} ; \mathbf{e}-\mathbf{h}$ correspond to coarse-grained stresses, $\sigma_{22}$ due to $\underline{\xi}^{d}=\xi^{d} \underline{e}_{2}$ and $\mathbf{i}-\mathbf{l}$ correspond to coarsegrained stresses, $\sigma_{33}$ due to $\underline{\xi}^{d}=\xi^{d} \underline{e}_{3}$

microtextural differences in transmitting the load. For scan A, the percolating path consists of what seems to be elongated grains, mostly in the clay phase, while for scan $\mathrm{B}$, the load-bearing path seems more granular, spatially dispersed and consisted of both inclusions and clay phases. This is further quantified using $g(r)$ of the percolating path as shown in Fig. 21. One general observation consistent with Fig. 21 indicates short-range nature of percolating stresses in scan A compared to long-ranged one for scan B. However, the results vary based on different realizations of spatial stiffness. These results are also consistent with earlier continuum mechanics-based homogenization approaches, and indeed the backbone of the molecular informed microporoelastic model of [41] where a selfconsistent homogenization scheme is used to model mature systems, e.g., scan B, implying an effective granular texture while for immature systems, e.g., scan A, a MoriTanaka homogenization scheme, implying a matrix-inclusion effective texture is employed. Furthermore, coarsegrained stresses, as shown in Figs. 22 and 23, suggest that at a coarse-graining length scale of $\lambda=50$ less noisy, multimodal response emerges highlighting the competition between different load-bearing phases.

In the future, calibration based on higher order cumulants can be considered for refinement of the results while relaxing conditions on, for example, constant ratio of $\epsilon_{i j}^{(n, t)}$ 
as discussed before. This would also require overcoming limitations of LEM in its current formulation in capturing the range of transversely isotropic behavior (see Eq. 16) and isotropic behavior beyond $v=0.25$ by examining comparable methods that have addressed such restrictions such as peridynamics [50] and elastic networks [43].

\section{Conclusions}

Utilizing advancements in high-performance computing and imaging techniques, a methodology to calibrate and to validate effective interaction potentials of the solid constituents of a highly heterogeneous porous solid is presented. The spatial variations of elasticity are shown to be necessary ingredients for capturing heavy-tails of measured indentation data. The measured indentation data and simulated effective poroelastic pore-pressure coefficients generally seem to follow a Stable distribution, a manifestation of interactions of wide underlying PDFs. Stress transmission highlights the distinct percolation paths in each scan due to underlying microtextural features, while stress coarse-graining delineates the highly heterogeneous nature of the materials examined and the challenges involved in defining a representative elementary volume for continuum-based analysis. The proposed framework provides new insights into the interplay of texture and effective behavior of real materials while paving the way for designing durable and sustainable materials with imposed effective mechanical behavior.

Acknowledgements This work was funded by X-Shale Hub: the Science and Engineering of Gas Shale, a collaboration between Shell, Schlumberger, and the Massachusetts Institute of Technology, enabled through MIT's Energy Initiative. Authors would like to acknowledge Vincent Richefeu (Universite Joseph Fourier), JeanYves Delenne (Montpellier SupAgro) and Saeid Nezamabadi (Universite de Montpellier) who provided the backbone of the LEM code used for simulations. FR would like to acknowledge the support of the ICoME2 Labex (ANR-11-LABX-0053) and the A*MIDEX projects (ANR-11-IDEX-0001-02) cofunded by the French program Investissements $d$ Avenir, managed by the French National Reseach Agency (ANR).

\section{References}

1. Abedi S, Slim M, Hofmann R, Bryndzia T, Ulm FJ (2016) Nanochemo-mechanical signature of organic-rich shales: a coupled indentation-EDX analysis. Acta Geotech 11:559-572

2. Abousleiman Y, Hull K, Han Y, Al-Muntasheri G, Hosemann P, Parker S, Howard C (2016) The granular and polymer composite nature of kerogen-rich shale. Acta Geotech 11(3):573-594

3. Affes R, Delenne JY, Monerie R, Radjai F, Topin V (2012) Tensile strength and fracture of cemented granular aggregates. Eur Phys J E Soft Matter 35:117
4. Aleksandrov K, Ryzhova T (1961) Elastic properties of rockforming minerals. II. Layered silicates. Bull USSR Acad Sci Geophys Ser 9:1165

5. Bathe KJ (2014) Finite element procedure. Prentice Hall, Upper Saddle River

6. Beneviste Y (1987) A new approach to the application of MoriTanaka's theory in composite materials. Mech Mater 6(2):147

7. Beran M (1968) Statistical continuum theories. Wiley, London

8. Beroz F, Jawerth L, Munster S, Weitz D, Broedersz C (2017) Physical limits to biomechanical sensing in disordered fibre network. Nat Commun 8:16096

9. Berryman J (2005) Bounds and self-consistent estimates for elastic constants of random polycrystals with hexagonal, trigonal, and tetragonal symmetries. J Mech Phys Solids 53(10):2141

10. Berthonneau J, Grauby O, Abuhaikal M, Pellenq RJM, Ulm FJ, Van Damme H (2016) Evolution of organo-clay composites with respect to thermal maturity in type II organic-rich source rocks. Geochim Cosmochim Acta 195:68-83

11. Biot M (1941) General theory of three-dimensional consolidation. J Appl Phys 12(2):155

12. Bousige C, Ghimbeu C, Vix-Guterl C, Pomerantz A, Suleimenova A, Vaughan G, Garbarino G, Feygenson M, Wildgruber C, Ulm FJ, Pellenq RJ, Coasne B (2016) Realistic molecular model of kerogen's nanostructure. Nat Mater 15:576-582

13. Budiansky B (1965) On the elastic moduli for some heterogeneous materials. J Mech Phys Solids 13:223

14. Christoffersen J, Mehrabadi M, Nemat-Nasser S (1984) A micromechanical description of granular material behavior. J Appl Mech 48(2):339-344

15. Constantinidies G, Ravi Chandran K, Ulm FJ, Van Vilet K (2006) Grid indentation analysis of composite microstructure and mechanics: principles and validation. Mater Sci Eng A 430(1-2):189-202

16. Deirieh A, Ortega J, Ulm FJ (2012) Nanochemomechanical assessment of shale: a coupled WDS-indentation analysis. Acta Geotech 7(4):271-295

17. Delafargue A, Ulm FJ (2004) Explicit approximations of the indentation modulus of elastically orthotropic solids for conical indenters. Int J Solids Struct 41:7351

18. Dimas L, Giesa T, Buehler M (2014) Coupled continuum and discrete analysis of random heterogeneous materials: elasticity and fracture. J Mech Phys Solids 63:481-490

19. Dormieux L, Kondo D, Ulm FJ (2006) Microporomechanics. Wiley, Chichester

20. Dormieux L, Molinary A, Kondo D (2002) Micromechanical approach to the behavior of poroelastic materials. J Mech Phys Solids 50:571

21. Ebrahimi D, Whittle A, Pellenq RJM (2014) Mesoscale properties of clay aggregates from potential of mean force representation of interactions between nanoplatelets. J Chem Phys 140:154309

22. Eshelby J (1957) The determination of the elastic field of an ellipsoidal inclusion, and related problems. Proc R Soc A 241:376

23. Gnedenko B, Kolmogorov A (1954) Limit distributions for sums of independent random variables. Addison-Wesley, Boston

24. Hantal G, Brochard L, Laubie H, Ebrahimi D, Pellenq JM, Ulm FJ, Cosane B (2014) Atomic-scale modeling of elastic and failure properties of clays. Mol Phys Int J Interface Chem Phys 112:1294

25. Herrmann H, Roux S (1990) Statistical models for the fracture of disordered media. Random materials and processes. Elsevier, New York

26. Hershey A (1954) The elasticity of an isotropic aggregate of anisotropic cubic crystal. J Appl Mech 21:226-240

27. Hill R (1965) A self-consistent mechanics of composite materials. J Mech Phys Solids 13(4):213-222 
28. Hubler M, Gelb J, Ulm FJ (2017) Microtexture analysis of gas shale by XRM imaging. J Nanomech Micromech 7(3):564

29. Johnson S (2009) The NLOPT nonlinear-optimization package. http://ab-initio.mit.edu/nlopt/

30. Kardar M (2007) Statistical physics of particles. Cambridge University Press, Cambridge

31. Katahara K (1996) Clay minerals elastic properties. In: Proceedings of the 66th SEG annual meeting expanded technical program abstracts

32. Kröner E (1958) Berechnung der elastischen Konstanten des Vielkristalls aus den Konstanten des Einkristalls. Z Phys 151(4):504-518

33. Larsson P, Giannakopoulos A, SoDerlund E, Rowcliffe D, Vetergaard R (1996) Analysis of Berkovich indentation. Int J Solids Struct 33:221

34. Laubie H, Monfared S, Radjai F, Pellenq RM, Ulm FJ (2017) Disorder-induced stiffness degradation of highly disordered porous materials. J Mech Phys Solids 106:207-228

35. Laubie H, Monfared S, Radjai F, Pellenq RM, Ulm FJ (2017) Effective potentials and elastic properties in the lattice-element method: isotropy and transverse isotropy. J Nanomech Micromech $7: 3$

36. Laubie H, Radjai F, Pellenq R, Ulm FJ (2017) Stress transmission and failure in disordered porous media. Phys Rev Lett 119:075501

37. Masoero E, Del Gado E, Pellenq RM, Ulm FJ, Yip S (2012) Nanostructure and nanomechanics of cement: polydisperse colloidal packing. Phys Rev Lett 109:3-6

38. Masoero E, Del Gado E, Pellenq RM, Ulm FJ, Yip S (2014) Nano-scale mechanics of colloidal C-S-H gels. Soft Matter 10:491

39. Mavko G, Mukerji T, Dvorkin J (2003) Rock physics handbook: tools for seismic analysis in porous media. Cambridge University Press, Cambridge

40. Monfared S, Laubie H, Radjai F, Pellenq RM, Ulm FJ (2017) Mesoscale poroelasticity of heterogeneous media. J Nanomech Micromech 7(4):04017016

41. Monfared S, Ulm FJ (2016) A molecular informed poroelastic model for organic-rich, naturally occurring porous geocomposites. J Mech Phys Solids 88:186

42. Mori T, Tanaka K (1973) Average stress in matrix and average elastic energy of materials with misfitting inclusions. Acta Metall 21:571

43. Norris A (2014) Mechanics of elastic networks. Proc R Soc A 470:20140522

44. Powell M (1994) A direct search optimization method that models the objective and constraint functions by linear interpolation. In: Gomez S, Hennart J-P (eds) Advances in optimization and numerical analysis. Kluwer Academic, Dordrecht
45. Powell M (1998) Direct search algorithms for optimization calculations. Acta Numer 7:287

46. Radjai F, Jean M, Moreau JJ, Roux S (1996) Force distribution in dense two-dimensional granular systems. Phys Rev Lett 77:274

47. Seewald J (2003) Organic-inorganic interactions in petroleumproducing sedimentary basins. Nature 426:327-333

48. Semnani SJ, Borja RI (2017) Quantifying the heterogeneity of shale through statistical combination of imaging across scales. Acta Geotech 12:1193-1205

49. Seo YS, Ichikawa Y, Kawamura K (1999) Stress-strain response of rock forming minerals by MD simulations. Mater Sci Res Int 5:13

50. Silling S, Lehoucq R (2010) Peridynamic theory of solid mechanics, pp. 87185-1322 . Tech. Rep. 1, Sandia National Laboratories, Albuquerque, New Mexico, USA

51. Suquet M (1987) Elements of homogenization for inelastic solid mechanics. In: Homogenization techniques for composite media. Springer, Berlin, pp 193-278

52. Tai K, Dao M, Suresh S, Palazoglu A, Ortiz C (2007) Nanoscale heterogeneity promotes energy dissipation in bone. Nat Mater 6:454

53. Tissot B, Welte D (1984) Petroleum formation and occurrence. Springer, Berlin

54. Topin V, Delenne JY, Radjai F, Brendel L, Mabille F (2007) Strength and failure of cemented granular matter. Eur Phys J E Soft Matter 23:413

55. Torquato S (2002) Random heterogeneous materials: microstructure and macroscopic properties. Springer, Berlin

56. Ulm FJ, Vandamme M, Bobko C, Ortega J, Tai K, Ortiz C (2007) Statistical indentation techniques for hydrated nanocomposites: concrete, bone, and shale. J Am Chem Soc 90(9):2677-2692

57. Vaughan M, Guggenheim S (1986) Elasticity of muscovite and its relationship to crystal structure. J Geophys Res 91:4657

58. Veneziano D (2003) Computational fluid and solid mechanics. Elsevier, Cambridge

59. Weibull W (1939) A statistical theory of the strength of materials. R Swed Inst Eng Res (Ingenors Vetenskaps Akadiens Handlingar) 151:1

60. Weibull W (1951) A statistical distribution function of wide applicability. J Appl Mech 18:293-297

61. Younis S, Kauffmann Y, Bloch L, Zolotoyabko E (2012) Inhomogeneity of nacre lamellae on the nanometer length scale. Cryst Growth Des 12(9):4574-4579

62. Zaoui A (2002) Continuum micromechanics: a survey. J Eng Mech 8:808 